Document downloaded from:

http://hdl.handle.net/10251/148012

This paper must be cited as:

Valera Fernández, A.; Díaz-Rodríguez, M.; Vallés Miquel, M.; Oliver, E.; Mata Amela, V.; Page Del Pozo, AF. (2017). Controller-observer design and dynamic parameter identification for model-based control of an electromechanical lower-limb rehabilitation system. International Journal of Control. 90(4):702-714. https://doi.org/10.1080/00207179.2016.1215529

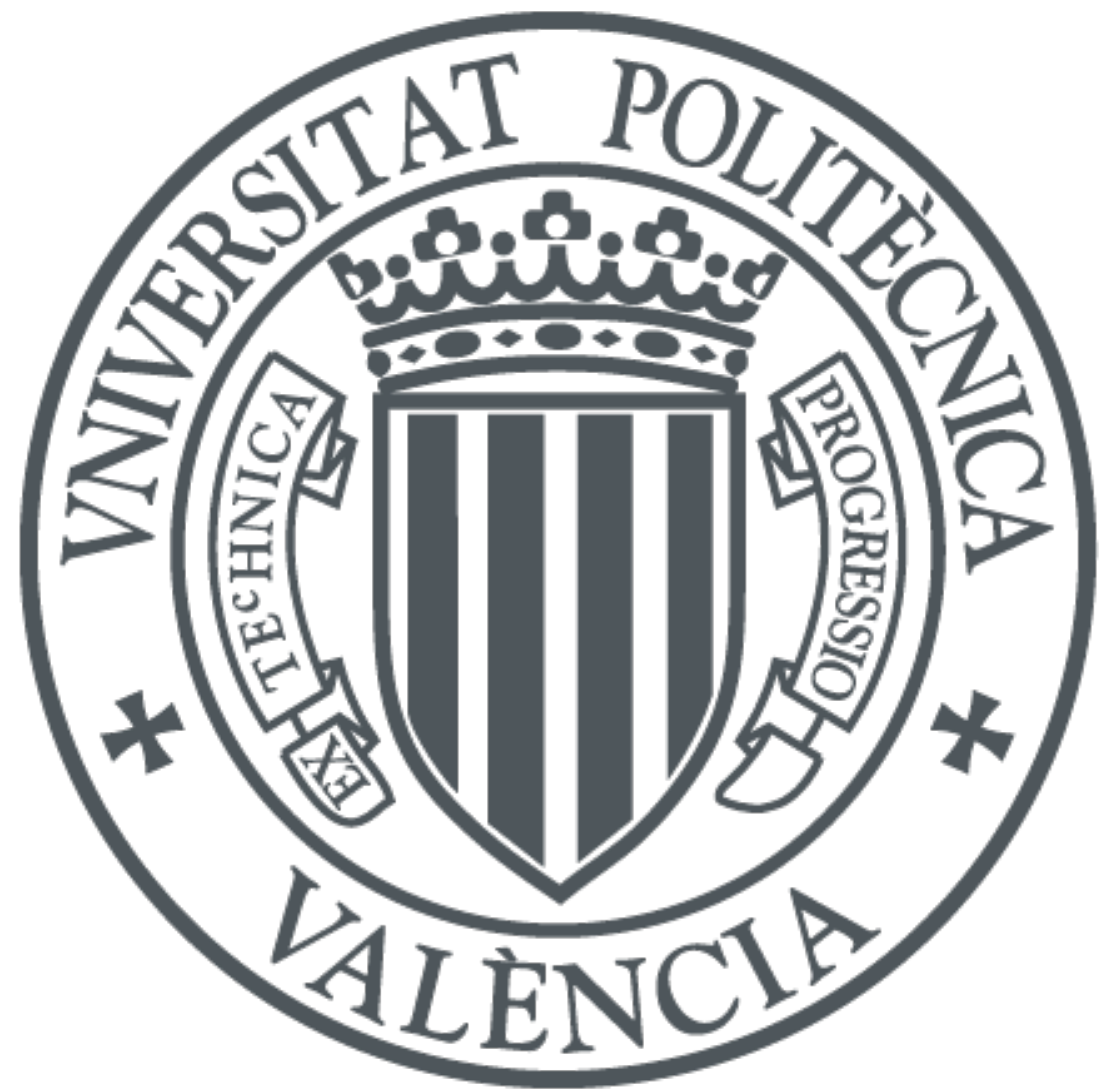

The final publication is available at

https://doi.org/10.1080/00207179.2016.1215529

Copyright Taylor \& Francis

Additional Information 


\section{Controller-observer design and dynamic parameter identification for model-based control of an electromechanical lower-limb rehabilitation system}

A. Valera ${ }^{(1)}$, M. Díaz-Rodríguez ${ }^{(2)}$, M. Valles ${ }^{(1)}$, E. Oliver $^{(1)}$, V Mata ${ }^{(3)}$, A. Page ${ }^{(4)}$

avalera@ai2.upv.es; dmiguel@ula.ve; mvalles@ai2.upv.es; eoliver@ai2.upv.es;

vmata@mcm.upv.es; Alvaro.page@ibv.upv.es

${ }^{(1)}$ Instituto Universitario de Automática e Informática Industrial, Universitat

Politècnica de València, Valencia, Spain.

${ }^{(2)}$ Departamento de Tecnología y Diseño, Facultad de Ingeniería, Universidad de los Andes, Mérida, Venezuela.

${ }^{(3)}$ Centro de Investigación de Tecnología de Vehículos, Universitat Politècnica de València, Valencia 46022, Spain.

${ }^{(4)}$ Departamento de Física Aplicada, Universitat Politècnica de València, Valencia 46022, Spain. 
Rehabilitation is a hazardous task for a mechanical system, since the device has to interact with the human extremities without the hands-on experience the physiotherapist acquires over time. A gap needs to be filled in terms of designing effective controllers for this type of devices. In this respect, the paper describes the design of a model-based control for an electromechanical lower-limb rehabilitation system based on a Parallel Kinematic Mechanism. A controller observer was designed for estimating joint velocities, which are then used in a hybrid position/force control scheme. The model parameters are identified by customizing an approach based on identifying only the relevant system dynamics parameters. Findings obtained through simulations show evidence of improvement in tracking performance compared with those where the velocity was estimated by numerical differentiation. The controller is also implemented in an actual electromechanical system for lower-limb rehabilitation tasks. Findings based on rehabilitation tasks confirm the findings from simulations.

Keywords: lower-limb rehabilitation; model-based control; parameter identification, controller-observer design, parallel kinematic mechanism 


\section{Introduction}

The design of electromechanical systems based on industrial robotics is continually expanding into other non-traditional fields, basically due to emerging needs and demands; for example, mechanical devices that meet human and social needs (Aleocer, Vela, Blanco, A Gonzalez, \& Oliver, 2012). Rehabilitation devices are one such kind whose design and implementation focus on reducing the physical therapist's work, increasing the length of rehabilitation exercises, gathering information on the status of the patient's recovery and also enabling tele-operated devices. It seems natural to replace rehabilitation tasks with a mechanical device that has some degree of autonomy, since its implementation can reduce the physical therapist's work; however, the available devices are still expensive and need appropriate protocols for therapy (Díaz, Gil, \& Sánchez, 2011). Moreover, rehabilitation is a hazardous task due to the fact that a mechanical system interacts with a living subject without the "experience" that the physiotherapist acquires over time. Thus, a gap remains to be filled in terms of designing effective controllers for this type of devices, specifically in terms of the mechanical system's ability to measure variables that can be used to learn the experience the task requires.

The first generation of rehabilitation robots used the same control strategies as industrial robots, based on trajectory control for passive systems, evolving into forces or torque control in active rehabilitation robots (Cao et al., 2014). The effectiveness of these simple strategies was limited, so new strategies have been developed by incorporating some adaptation to the active participation of patients, such the assisted-as-needed (AAN) rehabilitation strategies (Emken, Bobrow, \& Reinkensmeyer, 2005). In Bejarano 
et al. (2016) there is a review of the functional requirements that control systems need to meet in order to improve the efficiency of rehabilitation robots.

Most papers published in this area relate to gait rehabilitation devices, powered lowerlimb prostheses or exoskeletons for upper limb rehabilitation (Marchal-Crespo, \& Reinkensmeyer, 2009; Jimenez-Fabian, \& Verlinden, 2012; Ferreira, Reis, \& Santos, 2016). However, the use of Parallel Kinematic Mechanisms (PKM) in rehabilitation platforms is much more limited and has not received much attention.

In this respect, this paper presents a model-based control of a mechanical lower-limb rehabilitation system based on a PKM. The control scheme has the advantage of having a hybrid position/force control where the velocity of the mechanism joints is obtained through the design of a controller-observer. In addition, the model for the controller was identified by customizing an approach based on identifying only the relevant system dynamics parameters.

PKMs or parallel robots are the subject of numerous studies in the field of robotics. There is a vast amount of literature dealing with the implementation of control strategies based on different controls like PD/PID (Wu, Zhang, Li, \& Ouyang, 2002), fuzzy logic (Stan, Balan, Maties, \& Rad, 2009), computed torque control or inverse dynamic control (Yang, Huang, \& Han, 2012) and adaptive control (Cazalilla, Valles, Mata, Diaz-Rodriguez, \& Valera, 2014), to name but a few.

In the field of rehabilitation there have been some studies on applications of PKMs for ankle rehabilitation (Yoon, Ryu, \& Lim, 2006), (Saglia, Tsagarakis, Dai, \& Caldwell, 2013), (Janwal, Hussain, \& Xie, 2015). An overview of control strategies for assisted lower-limb rehabilitation was presented by Meng et al. (2015). For instance, position trajectory tracking control is important in the early stages of rehabilitation 
when "passive" mode is required. However, in order to consider the interaction between living subject and device, hybrid position/force or impedance controls are developed. Moreover, to encourage patients' active participation, bio-signals-based controls obtained by EMG were included in the control scheme.

One basic feature of the control strategies applied so far is the assumption that exact full state information, i.e. position and velocity, is available for feedback. In practice, this assumption can only partially be fulfilled in the case of velocity measurement. In most PKM applications velocity sensors are frequently omitted for reasons of cost, volume and weight (Klafter, Chmielewski, \& Negin, 1989) and if, for example, tachometers are used, their measurements are often contaminated with a considerable amount of noise (Berghuis, \& Nijmeijer, 1993).

The simplest way to solve the velocity feedback problem, also from an implementation point of view, is by first-order numerical differentiation of the accurate position signal. However, this approximation has some drawbacks, especially for low and high velocities (Canudas de Wit, \& Fixot, 1991). Moreover, the quantization effect that inherently goes with this approach can produce undesired oscillations in the robot joint response or even cause it to become unstable (Klafter, Chmielewski, \& Negin, 1989). Other methods for real-time velocity estimation include: filtered derivative, alphabeta trackers, and Kalman filtering. For example, (Janabi-Sharifi, Hayward, \& Chen, 2000) describe a class of adaptive finite impulse response (FIR) velocity estimation techniques for addressing the same problem and they are used to enhance the control of a haptic device.

In this sense, we focus on the motion control problem of a PKM using partial knowledge of the state variables, i.e. only position. A straightforward approach to this problem follows a two-step design procedure: 1. Construct an auxiliary dynamical 
system, known as an observer, driven by the available inputs and outputs, which reconstructs the missing state variable; 2 . Design a state-feedback controller and replace the missing state variables with those reconstructed by the observer.

In the case of linear systems, this two-step procedure can be conducted separately due to the separation principle (Kwakernaak, \& Sivan, 1972). However, in the case of the PKM considered in this paper, as it is a non-linear system this principle cannot be assumed. In this case, a fine tuning of the observer structure with respect to the underlying control concept is crucial (Berghuis, \& Nijmeijer, 1993). In this way, two practical requirements can be fulfilled. First, the observer can effectively exploit the structural properties of the controller, giving conceptually simple solutions to the velocity reconstruction problem. Second, useful stability and robustness properties can be provided for the controller-observer combinations. Both aspects are discussed in detail below.

The following section describes the PKM for lower-limb rehabilitation, then we present the dynamic parameter identification procedure. After that, we develop the observer design model-based control of the PKM, and the controller is implemented on an actual electromechanical/system for lower-limb rehabilitation tasks. Finally, the conclusions are presented.

\section{The Electromechanical Lower-limb Rehabilitation System}

In previous works, the authors have developed a PKM with 3 degree of freedom (DoF) (Vallés, Díaz-Rodríguez, Valera, Mata, \& Page, 2012). Figure 1-a shows the PKM as a lower-limb rehabilitation system which consists of three legs connecting the moving platform to the base. Each leg has a slider attached to the base by an actuated prismatic joint $(\underline{\mathrm{P}})$, a coupler connected to the slider by a passive rotational joint $(\mathrm{R})$ and to the platform by a passive spherical joint (S). Although the 3PRS PKM is affected by 
parasitic movements (Carretero et al., 2000), these are not considered relevant to the application, especially considering that the subject's leg will have additional degrees of motion that would prevent the possibility of damage caused by these small, uncontrolled parasitic motions.

The center of the moving platform of the PKM presents one translation (height) and two rotations (pitch and roll) as DoF. A load cell is installed at the platform's center so that the forces being applied to the subject can be measured (see Figure 1-b). Kinematic motion of the platform can be estimated through photogrammetry. The mechanism has already been tested for ankle rehabilitation tasks (Valles et al., 2015) within the research stream of methodologies for designing biomechatronic systems which is currently ongoing at Universitat Politècnica de València (https://mebiomec.ai2.upv.es/).

Aerotech BMS465 AH brushless servomotors and Aerotech BA10 power amplifiers provide direct-drive to the ball screws that give the linear motion to each prismatic joint $\left[q_{1}, q_{6}, q_{8}\right]$. These DC motors are equipped with incremental encoders with a resolution of 1000 pulses per revolution. A sinusoidally commutated brushless DC motor has the same linear relationship between current and torque as Cetinkunt's brush-type DC motor (2015). The torque current constant can be selected from the datasheet (https://Www.aerotech.com/media/854170/bms.pdf) or through experiments. According to the findings from (Valles, Díaz-Rodríguez, Valera, Mata, \& Page, 2012) the currenttorque relationship is linear for the prescribed operating range of the motors. The relationship was verified through experiments that were conducted.

An industrial PC has been used to implement the control architecture of the parallel robot. It is a high performance $4 \mathrm{U}$ Rackmount industrial system with 7 PCI slots and 7 ISA slots. It has a $2.5 \mathrm{GHz}$ Intel ${ }^{\circledR}$ Pentium ${ }^{\circledR}$ Core 2 Quad/Duo processor and 4 GB 
SDRAM. This architecture has the advantage of having an open control system, allowing us to design controllers that are capable of dealing with a hazardous task such as rehabilitation in which a mechanical system interacts with a living subject.

The industrial computer has 3 Advantech $^{\mathrm{TM}}$ data acquisition cards installed: 2 PCI-1720 cards and 1 PCL-833 card. The first of these supply the control action for each parallel robot actuator, providing four 12-bit isolated digital-to-analog outputs for the Universal PCI 2.2 bus, multiple output ranges $(0 \sim 5 \mathrm{~V}, 0 \sim 10 \mathrm{~V}, \pm 5 \mathrm{~V}, \pm 10 \mathrm{~V})$, programmable software and an isolation protection of 2500 VDC between the outputs and the PCI bus.

The PCL-833 card is used to obtain the actuators' positions. It is an ISA card that includes four 32-bits quadruple $\mathrm{AB}$ phase encoders and an on-board 8-bit timer with a wide range time-based selector.

In order to establish force control, the system has been equipped with the Delta SI-330-30 ATI sensor, which is capable of measuring forces and torques in the XYZ axes using a monolithic instrumented transducer. The maximum range of forces is $\pm 3700 \mathrm{~N}$ for $\mathrm{X}$ and $\mathrm{Y}$, and $\pm 10000 \mathrm{~N}$ for the $\mathrm{Z}$ axis. The maximum range of torque is $\pm 270 \mathrm{~N} \cdot \mathrm{m}$ for $\mathrm{X}$ and $\mathrm{Y}$, and $\pm 400 \mathrm{~N} \cdot \mathrm{m}$ for the $\mathrm{Z}$ axis.

The programming language used to control the mechanical system is $\mathrm{C}++$. The PC uses the Linux Ubuntu operating system, patched with Xenomai (a real-time kernel) to give it real-time characteristics. The real-time middleware Open RObot COntrol Software (OROCOS) combined with the Robot Operating System (ROS) middleware has been used in order to implement model-based control (Vallés, Cazalilla, Valera, Mata, Page, 2013).

\section{Dynamic Parameter Identification}

Effective model-based control of a mechanical system relies on the accuracy with which 
the model dynamic parameters are obtained, which is generally performed by identification techniques. Approaches for dynamic parameter identification were first introduced for Serial Kinematics Mechanism (SKM) about thirty years ago (Atkeson, An, \& Hollerbach, 1986; Gautier, \& Khalil, 1988), and then for PKMs about twenty years ago (Gautier, Khalil, \& Restrepo, 1995; Codourey, \& Burdet, 1997). Since then, a vast amount of literature has been published, but the field still remains open, for instance (Janot, Gautier, M., Jubien, \& Vandanjon, 2014; Janot, Vandanjon, \& Gautier, 2016) for SRs, and (Briot, \& Gautier, 2015) for PKM. One of the characteristics of an open research field, especially PKMs, is that each of these systems has particular characteristics that are difficult to include in order to develop a general method. For instance, the dynamic model can consider whether or not the layout of the mechanism presents symmetry. In addition, since a PKM has passive joints, friction in those joints adds another factor to take into account when identifying the model. In the end, the analyst has to customize general algorithms to the specific case he or she is dealing with.

As common ground in identification techniques, only a sub-set of parameters called Base Parameters (BP) can be identified by measuring joint torques and positions. Furthermore, only a smaller subset of essential parameters (EP) contributes significantly to the system dynamics (Pham, \& Gautier, 1991). Díaz-Rodríguez, Mata, Valera, Page (2010) proposed an approach that not only identifies the essential parameters, but also verifies whether or not the smaller subset of parameters are feasible. The smaller subset of essential and also feasible parameters was named relevant parameters. This approach tackles most of the specific characteristics when dealing with the identification of a particular case of PKM (a parallel manipulator), offering some hints so that the analyst can customize the identification model for the system being studied. Since the lowerlimb rehabilitation system is based on a PKM architecture, we have applied the Diaz- 
Rodriguez (2010) approach for identifying the relevant parameters. The following paragraphs summarize the specific details and results for the case of the electromechanical rehabilitation system.

\section{Linear model in Base Parameters}

By applying the Denavit-Hartenberg convention to the parallel manipulator, a set of 9 generalized dependent coordinates has been obtained, as can be seen in Figure 2 . The active generalized coordinates $q_{1}, q_{6}$ and $q_{8}$ are associated with the actuated prismatic joints $(\mathrm{P})$, and the passive generalized coordinates, $q_{2}, q_{7}$ and $q_{9}$ are associated with the revolute joints (R). The coordinates $q_{3}, q_{4}$ and $q_{5}$ only correspond to one of the spherical joints (S). The spherical joint has been modeled by means of three mutually perpendicular revolute joints. Those generalized coordinates are grouped in a vector $\boldsymbol{q}$.

The equation of motion of a PKM modeled through a set of dependent generalized coordinates can be written as follows,

$$
\operatorname{M}(\mathbf{q}, \varphi) \cdot \ddot{q}+C(\dot{q}, \dot{q}, \varphi) \cdot \dot{q}+g(q, \varphi)=\varsigma-J^{T} \cdot \lambda
$$

where $\dot{\boldsymbol{q}}, \ddot{\boldsymbol{q}}$ are the generalized velocities and accelerations, $\boldsymbol{M}$ is the mass matrix of the system, $\boldsymbol{C}$ the matrix of centrifugal and Coriolis terms, $\boldsymbol{g}$ the vector of gravitational terms, $\boldsymbol{\varsigma}$ the vector of generalized forces, $\boldsymbol{J}$ the Jacobian matrix obtained by deriving the constraint equations with regard to all the generalized coordinates and $\lambda$ the vector of Lagrange multipliers. The vector $\boldsymbol{\varphi}$ includes all the inertial terms of the mobile links of the PKM, so that, for the $i$-th mobile link, the mass $m_{i}$, the first moment of masses $m x_{i}, m y_{i}, m z_{i}$ and the terms of the inertia matrix $I x x_{i}, I y y_{i}, I z z_{i}, I x y_{i}, I x z_{i}, I y z_{i}$, are all expressed with regard to local and non-centroidal reference systems, which are located 
at the joint of each link.

For identification purposes, the last term of equation (1), the generalized internal forces, is inconvenient, so it could be avoided by multiplying both terms of the equation (1) by an orthogonal complement (García de Jalón and Bayo, 1991), $\boldsymbol{R}$. In such a way, the equation of motion written in a suitable way for identification purposes can be obtained,

$$
R^{T} \cdot \mathbf{M}(\mathbf{q}, \varphi) \cdot \ddot{q}+R^{T} \cdot C(q, \dot{q}, \varphi) \cdot \dot{q}+R^{T} \cdot g(q, \varphi)=R^{T}
$$

Now considering the relationship between all the generalized coordinates and the active ones, equation (3) can be re-written as follows,

$$
\mathbf{M}_{F x \boldsymbol{F}}^{*}(\mathbf{q}, \boldsymbol{\varphi}) \cdot \ddot{\boldsymbol{q}}_{\boldsymbol{F} x \mathbf{1}}^{*}+\mathbf{C}_{\boldsymbol{F} x \boldsymbol{F}}^{*}(\boldsymbol{q}, \dot{\boldsymbol{q}}, \boldsymbol{\varphi}) \cdot \dot{\boldsymbol{q}}_{\boldsymbol{F} x \mathbf{1}}^{*}+\mathbf{g}_{F x 1}^{*}(\boldsymbol{q}, \boldsymbol{\varphi})=\boldsymbol{\zeta}_{\boldsymbol{F} x \boldsymbol{F}}^{*}
$$

$F$ being the number of degrees of freedom of the parallel robot, and the new vectors $\dot{\boldsymbol{q}}^{*}, \ddot{\boldsymbol{q}}^{*}$ correspond to the active generalized velocities and accelerations. From equation (3), the rigid body dynamics can be written as linear in the so-called base parameters (Gautier and Khalil, 1988),

$$
W\left(\mathbf{q}_{i}, \hat{\mathbf{q}}_{\mathrm{i}}, \ddot{\mathbf{q}}_{\mathrm{i}}\right) \cdot \boldsymbol{\varphi}=\mathbf{S}_{\mathbf{i}}^{*}
$$

where $\boldsymbol{W}\left(\boldsymbol{q}_{i}, \dot{\boldsymbol{q}}_{i}, \ddot{\boldsymbol{q}}_{i}\right)$ is the observation matrix, which is a function of the generalized coordinates, the velocities and the accelerations for the $i$-th pose of the PKM. For the robot considered, equation (4) constitutes an undetermined linear system; however, over a prescribed trajectory, equation (4) can be applied to a set of $n$ poses in order to build an overdetermined linear system. For a general mechanical system, the columns of the new observation matrix, corresponding to each robot pose, are not independent ones because some of the inertial parameters have no effect on the dynamic behavior of the 
system or they affect in linear combination. A set of base parameters (Gautier, 1991) has to be found such that,

$$
\boldsymbol{W}(\boldsymbol{q}, \dot{\boldsymbol{q}}, \ddot{\boldsymbol{q}}) \cdot \boldsymbol{\varphi}_{B}=\tau
$$

where $\boldsymbol{W}$ is now the global observation matrix, $\boldsymbol{\tau}$ the vector collecting the mapped generalized forces for the different robot poses, and $\boldsymbol{\varphi}_{\boldsymbol{B}}$ the vector including linear combinations of the inertial parameters grouped in $\boldsymbol{\varphi}$. Then, the dynamic base parameters can be found through Least Squares (LS) or Weighted Least Squares (WLS) as follows,

$$
\left[\left(W^{T} \cdot S \cdot W\right)^{-1} \cdot W^{T} \cdot S\right] \cdot \varphi_{B}=\tau
$$

where $\boldsymbol{S}$ is a suitable weight matrix computed from the standard deviation of measured torques. The size of the minimal combination for $\boldsymbol{\varphi}_{B}$ depends on the kinematic topology of the mechanism. The relationship can be found using the SVD technique (Gautier, 1991) as follows,

$$
\varphi_{B}=\varphi_{a}+B \cdot \varphi_{B}=\tau
$$

where $\boldsymbol{\varphi}_{\boldsymbol{a}}$ and $\boldsymbol{\varphi}_{\boldsymbol{b}}$ are subsets of $\varphi$ and $B$ is a matrix obtained after applying the SVD technique to matrix $\boldsymbol{W}$.

The linear combination $\varphi_{B}$ can be generated in different ways depending on the subset of parameters $\boldsymbol{\varphi}_{\boldsymbol{b}}$ that are selected. The approach proposed by Gautier (1991) for serial robots suggests that $\boldsymbol{\varphi}_{\boldsymbol{b}}$ should be selected by starting with the first row of the subset of the matrix providing a linear relationship of the parameters; this approach has been implemented in the open source OpenSYMORO packages (Khalil et al., 2014). Diaz-Rodriguez (2010) noted that the selection of $\boldsymbol{\varphi}_{\boldsymbol{b}}$ affects the conditioning of 
matrix $\boldsymbol{W}$. In order to reduce the impact of noise in the parameters solution, we have to search for the set of parameters that provides the lowest condition number. As an example, we present here two of the possible combinations for the 3 DOF PKM, set 1:, $\left[m_{1}, m_{2}, m_{3}, m_{4}, m_{5}, m_{6}\right]$, and set 2: $\left[m_{1}, I y y_{3}, m_{3}, m x_{3}, m_{5}, m_{7},\right]$. The sampling trajectories for computing the condition number of each matrix $\boldsymbol{W}$ with regard to the set of parameters were obtained using the optimal trajectory approach based on the goal attainment method (Díaz-Rodríguez, Iriarte, Mata, \& Ros, 2009). The mean value for the condition number using subset 1 was about 50, and for subset 2 it was about 110 . Therefore, we selected subset 1 for identification purposes.

In addition to rigid body dynamics, additional coefficients for friction modeling are included in the identification process; the condition is that those models are linear in the coefficients to be identified. It must be mentioned that before assembling the PKM, each actuator was tested independently and it was apparent that the improvement of using a non-linear friction model did not justify its application compared with the benefits of keeping the whole identification model linear in parameters (Farhat, Diaz, \& Mata, 2007). The selected friction model provides the $j$-th joint friction torque function considering two parameters

$$
\tau_{f_{j}}=F_{c} \cdot \operatorname{sign}\left(\dot{q}_{j}\right)+F_{v} \cdot \dot{q}_{j}
$$

where $F_{c}$ represents the coefficient of Coulomb friction, and $F_{v}$ viscous friction for each joint. Equation (8) is applied only to the actuated joints. It is important to point out that although friction may not be relevant at low speeds, the type of linear actuator used in this PKM has a high Coulomb friction component, and considering that sudden changes in the sign of the actuator motion occur during a rehabilitation path, that friction component cannot be ignored. 


\section{Physical Feasibility}

The 3-DOF PKM consists of three legs in a symmetrical layout. Due to manufacturing and assembly methods, it is difficult for the legs to be identical; however, the differences can be considered small, so their contribution to the system dynamics will remain small. We considered having identical leg geometry as a valid assumption. Therefore, $m_{1}=m_{4}=m_{6}, \boldsymbol{I}_{G_{2}}=\boldsymbol{I}_{G_{5}}=\boldsymbol{I}_{G_{7}}, \boldsymbol{I}_{G_{i}}$ being the inertia matrix with regard to a reference system attached to the $i$-th bar and located in the center of gravity and $m y_{2}=m y_{5}=m y_{7} \approx 0$. Moreover, if we also consider that the geometry of the platform is cylindrical with a small width, we can obtain the parameters shown in Table 1.

Table 1 shows the subset of relevant parameters $\varphi_{\boldsymbol{B}}$, before applying the reduction process considering feasibility. In the table, $V_{r}$ is the distance $B_{i}, P_{i}$ from the spherical to the revolution joint (see Figure 2), $l_{m}$ is the distance $P_{i}, P_{j}$ between two adjacent spherical joints at the platform, $a=l_{m} \cdot \sin \left(120^{\circ}\right), b=l_{m} \cdot \cos \left(120^{\circ}\right)$, and $c=l_{m}$. From $\boldsymbol{\varphi}_{\boldsymbol{B}}, m_{3}$ can be obtained given the values of $m_{1}$ and $m_{2}$. After that, the remaining parameters can be found with respect to the values of the three masses and the identified parameters grouped in vector $\boldsymbol{\varphi}$. The equation of feasibility can be written as

$$
\begin{aligned}
& \boldsymbol{I}_{G_{2}}=f\left(\varphi, m_{1}, m_{2}\right)>0 \\
& \boldsymbol{I}_{G_{3}}=f\left(\varphi, m_{1}, m_{2}\right)>0 \\
& m_{3}=f\left(\varphi, m_{1}, m_{2}\right)>0
\end{aligned}
$$

In order to solve the above equations, we can define lower and upper bounds of $m_{1}$ and $m_{2}$ so that at each step in the reduction process we can search at least for a discrete value within the bounds, so that we are able to find a set of feasible $\boldsymbol{I}_{G_{2}}, \boldsymbol{I}_{G_{3}}, m_{3}$. Based on the geometry of the link and the density of the material, we set the bounds for 
the platform mass $m_{3}$ to [ 515$] \mathrm{kg}$ and [0.1 5 [ $\mathrm{kg}$ for $m_{1}$ and $m_{2}$, respectively. Friction parameters are considered positive values only, as is the rotational inertia of the screw driving system.

The identification process starts by measuring positions and torques when the PKM follows an optimal trajectory. Then, equation (5) and (8) are used to obtain the regressor matrix $\boldsymbol{W}$, and after that the relevant parameters are found through equation (2). At this point we check whether the parameters are feasible or not, so that we can decide whether to reduce our model. The process continues until a model with a feasible parameter is found.

\section{Observer Design for Model-Based Control}

\section{Model-Based Position Control}

The velocity observer design problem consists of constructing an auxiliary dynamic system that asymptotically reconstructs the velocity signal from input-output measurements, i.e. torque ( ) and position ( $\boldsymbol{q})$, respectively. A number of strategies have been developed to tackle this design problem. For instance, (Canudas de Wit, \& Slotine, 1991) present sliding mode velocity observers for industrial robots, which are based on the concept of variable structure systems.

One useful feature of these observers is their good robustness to uncertainties in rigid body dynamics, which is caused by the presence of switching terms in the observer dynamics. Unfortunately, these switching terms also generate high-frequency chattering in the velocity estimate, which in a closed loop may produce undesirable effects such as excitation of unmodeled high-frequency dynamics and fatigue of system actuators. This is a major practical drawback of sliding mode observers. The observer design proposed by (Berghuis, Löhnberg, \& Nijmeijer, 1991) starts as follows: 


$$
\left\{\begin{array}{l}
\frac{d}{d t} \widehat{\boldsymbol{q}}=\mathbf{z}+\boldsymbol{L}_{\boldsymbol{d}} \cdot \widetilde{\boldsymbol{q}} \\
\frac{d}{d t} \boldsymbol{z}=\boldsymbol{M}^{-\mathbf{1}}(\boldsymbol{q}, \boldsymbol{\theta}) \cdot\left(\boldsymbol{\tau}-\boldsymbol{C}\left(\boldsymbol{q}, \dot{\boldsymbol{q}}_{\mathbf{0}}, \boldsymbol{\theta}\right) \cdot \dot{\boldsymbol{q}}_{\mathbf{0}}-\boldsymbol{G}(\boldsymbol{q}, \boldsymbol{\theta})+\boldsymbol{L}_{\boldsymbol{p} \mathbf{1}} \cdot \widetilde{\boldsymbol{q}}\right)+\boldsymbol{L}_{p 2} \cdot \widetilde{\boldsymbol{q}}
\end{array}\right.
$$

where $\left[\widehat{\boldsymbol{q}}^{\boldsymbol{T}} \boldsymbol{z}^{\boldsymbol{T}}\right]$ is the observer state, $\dot{\hat{\boldsymbol{q}}}$ represents the estimated velocity, $\widetilde{\boldsymbol{q}} \equiv \boldsymbol{q}-\widehat{\boldsymbol{q}}$ is the observer position estimation error, $\boldsymbol{L}_{d}=\boldsymbol{L}_{d}^{T}>0, \boldsymbol{L}_{p 1}=\boldsymbol{L}_{p 1}^{T} \geq \mathbf{0}, \boldsymbol{L}_{p 2}=\boldsymbol{L}_{p 2}^{T} \geq \mathbf{0}$ and $\dot{\boldsymbol{q}}_{0}=\dot{\hat{\boldsymbol{q}}}-\boldsymbol{\Lambda}_{2} \widetilde{\boldsymbol{q}}$ with $\boldsymbol{\Lambda}_{2}=\boldsymbol{\Lambda}_{2}^{T}$.

The computed torque controller can be defined as

$$
\begin{aligned}
& \tau_{c}=M(q, \theta) \cdot a+C(q, \dot{\hat{q}}, \theta) \cdot \dot{\hat{q}}+G(q, \theta) \\
& a=\ddot{q}_{d}-K_{d} \cdot\left(\dot{\hat{q}}-\dot{q}_{d}\right)-K_{p} \cdot e
\end{aligned}
$$

where $\dot{\hat{\boldsymbol{q}}}$ represents the velocity estimate. This estimate is obtained/from the nonlinear open-loop observer. In order to tune the observer more finely with respect to this control strategy, assume $\boldsymbol{L}_{\boldsymbol{p}}=0$ and $\boldsymbol{\Lambda}_{2}=0$. Then the observer systems become:

$$
\begin{aligned}
& \frac{d}{d t} \widehat{\boldsymbol{q}}=\mathbf{z}+\boldsymbol{L}_{\boldsymbol{d}} \cdot \widetilde{\boldsymbol{q}} \\
& \frac{d}{d t} \boldsymbol{z}=\boldsymbol{M}^{-\mathbf{1}}(\boldsymbol{q}, \boldsymbol{\theta}) \cdot(\boldsymbol{\tau}-\boldsymbol{C}(\boldsymbol{q}, \dot{\hat{\boldsymbol{q}}}, \boldsymbol{\theta}) \cdot \dot{\boldsymbol{q}}-\boldsymbol{G}(\boldsymbol{q}, \boldsymbol{\theta}))+\boldsymbol{L}_{\boldsymbol{p} 2} \cdot \widetilde{\boldsymbol{q}}
\end{aligned}
$$

Combining these equations:

$$
\begin{aligned}
& \text { Controller }\left\{\begin{array}{l}
\boldsymbol{t}_{\boldsymbol{c}}=\boldsymbol{M}(\boldsymbol{q}, \boldsymbol{\theta}) \cdot \boldsymbol{a}+\boldsymbol{C}(\boldsymbol{q}, \dot{\hat{\boldsymbol{q}}}, \boldsymbol{\theta}) \cdot \dot{\hat{\boldsymbol{q}}}+\boldsymbol{G}(\boldsymbol{q}, \boldsymbol{\theta}) \\
\boldsymbol{a}=\ddot{\boldsymbol{q}}_{\boldsymbol{d}}-\boldsymbol{K}_{\boldsymbol{d}} \cdot\left(\dot{\hat{\boldsymbol{q}}}-\dot{\boldsymbol{q}}_{\boldsymbol{d}}\right)-\boldsymbol{K}_{\boldsymbol{p}} \cdot \boldsymbol{e}
\end{array}\right. \\
& \text { observer }\left\{\begin{array}{l}
\dot{\hat{\boldsymbol{q}}}=\boldsymbol{z}+\boldsymbol{L}_{\boldsymbol{d}} \cdot \widetilde{\boldsymbol{q}} \\
\dot{\boldsymbol{z}}=\boldsymbol{a}+\boldsymbol{L}_{\boldsymbol{p} 2} \cdot \widetilde{\boldsymbol{q}}
\end{array}\right.
\end{aligned}
$$

An interesting feature of the observer part is that it is rendered linear by the control input, which is a consequence of the feedback linearizing property of the computed torque method. To explain this, remember that the computed torque controller results in the linear system: $\ddot{\boldsymbol{q}}=\boldsymbol{a}$ 
For the stability analysis, define $\boldsymbol{y}^{\boldsymbol{T}}=\left[\begin{array}{llll}\dot{\overline{\boldsymbol{q}}}^{\boldsymbol{T}} & \overline{\boldsymbol{q}}^{\boldsymbol{T}} & \dot{\tilde{\boldsymbol{q}}}^{\boldsymbol{T}} & \widetilde{\boldsymbol{q}}^{\boldsymbol{T}}\end{array}\right]$ where the observer tracking error $\overline{\boldsymbol{q}} \equiv \boldsymbol{e}-\widetilde{\boldsymbol{q}}\left(=\widehat{\boldsymbol{q}}-\boldsymbol{q}_{\boldsymbol{d}}\right)$, and choose as a Lyapunov function candidate a function that consists of two separate parts: one for the observer tracking error dynamics and another for the observer error dynamics (Berghuis, Löhnberg, \& Nijmeijer, 1991). With this function the closed-loop system is locally exponentially stable.

Through simulations, the following figures show the PKM response obtained with the controller-observer for the first joint. Figure 3 shows the reference and the $q_{1}$ position. Figure 4 shows the velocity and its estimation. The results point to the conclusion that the controller was able to generate very good tracking performance. Notice that the mass matrix, centrifugal and gravitational terms of equation (10) can be obtained in terms of identified relevant parameters.

Figure 5 shows the error between the PKM velocity and the velocity estimation. The velocity estimation is also very good, providing a mean error of $0.372 \mathrm{~mm} / \mathrm{s}$.

As was mentioned in the Introduction, velocity sensors may not be available in the electromechanical system, and even when they are available, noise is usually a problem, as is the case with tachometers. Sensor noise places limits on controller gains, resulting in larger tracking errors. 
Joint velocities can be determined by first-order differentiating position measurements obtained with a potentiometer or optical encoders. Usually, an optical encoder has a large signal-to-noise ratio; with differentiation, however, even low levels of noise in the position signal may produce unacceptably large velocity noise.

In order to verify this, using the same PKM model and the same inverse dynamics controller with the same gain values, two simulations have been executed. In the first simulation the velocity estimation is obtained by first-order differentiating position measurements. In the second, the velocity observer has been used.

The position responses of both controllers are very similar, obtaining good trajectory tracking performance. However, there are differences in the control actions obtained in these simulations. Figure 6 shows the control actions (torque) applied to the first joint of the PKM.

Note the low level of chattering obtained with the velocity observer compared with the estimation calculated by first-order differentiating positions, which reduces the possibility of exciting unmodeled higher frequency dynamics of the PKM. Another advantage of this reduced chattering in the action control is less mechanical wear and lower energy consumption.

To verify experimentally the efficiency of the velocity observer with respect to the first-order position differentiation, these controllers have been implemented and tested in the actual electromechanical system shown in Section 2 (Figure 1a).

Figure 7 shows the reference and the $q_{1}$ position. Figure 8 shows the velocity estimations, and Table 2 shows the results of the comparison between the different 
approaches. We have compared: the generalized joint coordinate $q_{1}$, and its velocity. The results obtained with the remaining active coordinates $\left(q_{6}\right.$ and $\left.q_{8}\right)$ are similar.

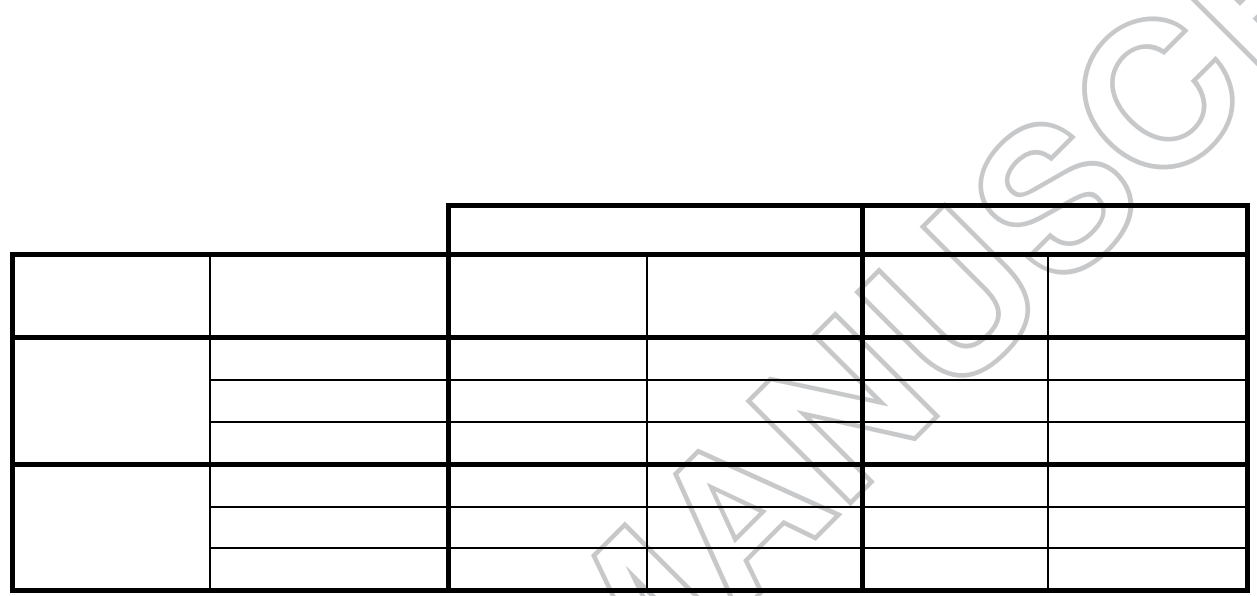

As regards the position variable, we calculated the difference with the reference value and the actual PKM joint $q_{1}$. This difference is described by three parameters: i) the mean error, which quantifies the bias; ii) the std of the error, which quantifies the error amplitude around the reference value and; iii) the average lag between the real variable and reference, which evaluates the phase error. This value was calculated as described in (Ramsay \& Silverman, 2006), $180^{\circ}$ corresponding to the value of two signals in antiphase. The variables mean of error and std of error are expressed in both absolute magnitude and as a percentage of the signal range.

With respect to the derivatives, we have compared the estimated velocities with the derivative of the reference variable. This derivative has been calculated using a smoothing procedure based on B-splines bases (Ramsay and Silverman, 2006). The optimal level of smoothing was defined according to the criteria of no correlation of 
residuals (Page et al., 2006). The comparison parameters are the same as in the case of the variable $q_{1}$.

As shown in the table, the two methods give a very low mean error, which means that the system works without bias, as regards both the position variable and its derivative. However, there are differences in terms of the oscillations around the error value and in the phase error. Thus, for the FOD estimation the error is about twice that found with the Observer method. A larger lag is also seen, although both/are practically negligible.

The differences are much more pronounced for the velocities. Indeed, in the case of the FOD method, the error around the reference is $7.29 \%$ of the amplitude, but when the Observer estimation is used, this error decreases to $1.98 \%$. The differences are also very pronounced with respect to the phase error, which is three times higher for the FOD method.

\section{Hybrid Force/Position Control}

Industrial controllers support the development of automation programs that perform a sequence of movements. Due to their versatility, PKMs are quite a flexible solution. However, while standard PKM motions are position controlled only, force control permits flexible adaptation with respect to inaccuracies in human interaction.

As mention before, the PKM for lower-limb rehabilitation has three degrees of freedom: the height $(\mathrm{z})$ of the platform and the pitch and roll orientation ( and ). The hybrid force/position control of Figure 9 has been developed for the PKM. Conceptually, the control scheme is based on the work of Raibert and Craig (1981). The PKM controls the force applied by the platform to the environment considering the force reference $\boldsymbol{f}_{\boldsymbol{d}}$. For the position/orientation control, the scheme uses the Cartesian references $\left[\boldsymbol{z}_{d}, \gamma_{d}, \boldsymbol{\beta}_{d}\right]^{T}$. 
In this architecture, the position references $\boldsymbol{q}_{\boldsymbol{d}}$ are computed and sent to the joint space system controller (“Position Controller” block). These references are obtained from the output of the "Inverse Kinematics" block $\left(\widehat{\boldsymbol{q}}_{\boldsymbol{d}}\right)$ and $\Delta \boldsymbol{q}$, the output of the integral of the Jacobian function.

For the calculation of $\Delta \boldsymbol{q}$, a force control algorithm is programmed. In this work it is an explicit PID force control that uses the error signal between the force reference $\boldsymbol{f}_{\boldsymbol{d}}$ and the force measured on the mobile platform $\boldsymbol{f}$ :

$$
\boldsymbol{F}=\boldsymbol{K}_{\boldsymbol{p}} \cdot\left(\boldsymbol{f}_{\mathrm{d}}-\boldsymbol{f}\right)+\boldsymbol{K}_{\boldsymbol{I}} \cdot \int\left(\boldsymbol{f}_{\mathrm{d}}-\boldsymbol{f}\right) \cdot \mathrm{dt}+\boldsymbol{K}_{\boldsymbol{d}} \cdot \frac{\boldsymbol{d}}{\mathrm{dt}}\left(\boldsymbol{f}_{\mathrm{d}}-\boldsymbol{f}\right)(15)
$$

The effect of the three parameters of a PID controller is well known and has been widely addressed in the literature (Aström, \& Murray, 2010). The integral term assures zero tracking error. The function of the derivative term is to damp the system.

Finally, the position controller compares the $\boldsymbol{q}_{d}$ references and the PKM joint positions $\boldsymbol{q}$ and calculates the control actions to apply to the system. These control actions are obtained by means of the controller-observer based on the feedback linearization of equations (13-14).

The following figures show the system response for the hybrid force/position control. The pitch orientation is based on the walking motion reference for the gait cycle and it corresponds to dorsiflexion/plantar flexion ankle movements. The roll orientation corresponds to the reference for eversion/inversion ankle movements. In this case, the reference for this roll angle is 0 . Finally, the reference force corresponds to the force when the human foot is resting on the ground. These Cartesian references are based on (Yoon, Ryu, \& Lim, 2006).

Figure 10 shows the reference and the PKM response for the pitch orientation, and Figure 11 represents the roll orientation. The position control results present very 
good tracking performance. Figure 12 shows the force control results and, as in the previous cases, the response was also very good.

\section{Lower-Limb Exercises with Electromechanical Rehabilitation Systems}

In order to rehabilitate or strengthen injured lower limbs, different exercises can be performed with the PKM proposed in this work. These exercises can be passive or active. Passive exercises are performed without any voluntary movement by the patient, while active exercises are performed with voluntary movement by the patient. In order to show how the mechanism operates, a series of exercises have been performed with healthy subjects.

In passive exercises, the mechanism is programmed to follow a specific position reference prescribed by a specialist. Thus, using the PKM, a number of references have been generated to rehabilitate the lower limbs. In this case, the forces and torques applied to the ankle are being monitored all the time in order to avoid dangerous overexertion.

In active exercises, there are several types of active movements, such as activeresistive or active-assistive exercises. In the first case (strengthening or resistive exercises) the patient has to overcome a resistance imposed by the specialist. Activeassistive exercises are usually done at an early stage in the rehabilitation process where, for example, the patient is not able to carry out the movement against gravity by him or herself. For this reason, external help is needed to perform them correctly.

In this work, different rehabilitation exercises have been proposed using the PKM and its open control unit. The position controller has been developed using the inverse dynamics control algorithm of equation (13). Because the PKM has no velocity sensors, the velocity observer of equation (14) was also implemented. The controllerobserver operates at a frequency of $100 \mathrm{~Hz}\left(\mathrm{~T}_{\mathrm{s}}=10 \mathrm{~ms}\right)$. The following figures show the 
response for an active-resistive application. The aim is to keep the platform of the parallel manipulator in a horizontal position by applying opposed torques to the motion of the platform.

For this exercise there are two stages. In the first one (from $t=0 \mathrm{~s}$ to $9 \mathrm{~s}$ ) a position control is established. There is a smooth movement reference to position the PKM platform at the height $(0.144 \mathrm{~m})$ required by the physiotherapist. The second stage (from $t=9 \mathrm{~s}$ to $90 \mathrm{~s}$ ) establishes a force control. The force reference is a low-frequency sinusoidal (pi/2 rad/s) force reference, with an amplitude of $60 \mathrm{~N}$. The parameters of the force reference are chosen by the physiotherapist according to the patient's condition.

Figure 13 shows the reference and the force applied by the patient. In this exercise, the patient can follow the force indicated by the therapist without any problem.

According to the force error and the platform orientation reference, the joint references are calculated in real time by the Jacobian of the mechanism. Figure 14 shows the reference and the response for the first joint of the system. Because the position control is a model-based controller, it presents a very good response, with a very small error (see Figure 15).

\section{Conclusions}

This paper has presented a controller-observer model-based control design for an electromechanical lower-limb rehabilitation system. The control scheme is based on a model-based controller that uses the dynamic parameters obtained by means of an identification process. Because it is assumed that there are no velocity sensors, the controller has a velocity observer to obtain its estimation.

The controller-observer has been tested in simulations and in an actual electromechanical system: a PKM. The control unit has an open architecture based on an industrial PC running open software and control middleware: OROCOS and ROS. 
This control architecture has several advantages: it is a very economical system and it enables different control strategies to be implemented using different sensors, such as potentiometers, force sensors and machine vision cameras, to name but a few. In order to demonstrate the performance of the developed system, a hybrid position/force scheme has been also developed. This scheme makes it possible to carry out different lower-limb rehabilitation exercises.

Finally, the velocity observer results in a smooth implementation, which reduces the possibility of exciting unmodeled higher frequency dynamics of the electromechanical system, reducing the chattering in the applied control torque and leading to less mechanical wear and lower energy consumption.

\section{Disclosure statement}

The authors declare that the research was conducted in the absence of any commercial, financial or personal relationships that could be construed as a potential conflict of interests.

\section{Funding}

This work was partially financed by the Plan Nacional de $I+D$, Comision Interministerial de Ciencia y Tecnología (FEDER-CICYT) under the project DPI201344227-R and by the Instituto U. de Automática e Informática Industrial (ai2) of the Universitat Politècnica de València.

\section{References}

Alcocer, W., Vela, L., Blanco, A., Gonzalez, J., \& Oliver, M. (2012). Major trends in the development of ankle rehabilitation devices. Dyna, 79(176), 45-55.

Aström, K., \& Murray, R. (2010). Feedback Systems: An Introduction for Scientists and Engineers. Princeton University Press. 
Atkeson, C. G., An, C. H., \& Hollerbach, J. M. (1986). Estimation of inertial parameters of manipulator loads and links. The International Journal of Robotics Research, 5(3), 101-119.

Bejarano, N. C., Maggioni, S., De Rijcke, L., Cifuentes, C. A., \& Reinkensmeyer, D. J. (2016). Robot-Assisted Rehabilitation Therapy: Recovery Mechanisms and Their Implications for Machine Design. In Emerging Therapies in Neurorehabilitation II (pp. 197-223). Springer International Publishing.

Berghuis, H., Löhnberg, P., \& Nijmeijer, H. (1991) Tracking control of robots using only position measurements. In 30th IEEE Conference on Decision and Control, pp. 1039-1040.

Berghuis, H., \& Nijmeijer, H. (1993). A Passivity Approach to Controller-Observer Design for Robots. IEEE Trans. on Robotics and Automation, 9(6), 740-754.

Briot, S., \& Gautier, M. (2015). Global identification of joint drive gains and dynamic parameters of parallel robots. Multibody System Dynamics, 33(1), 3-26.

Canudas de Wit, C., \& Fixot, N. (1991). Robot Control via Robust Estimated State Feedback. IEEE Trans. on Automatic Control, 36, 1497-1501.

Canudas de Wit, C., \& Slotine, J.J.-E. (1991). Sliding Observers for Robot Manipulators. Automatica, 27, 859-864.

Cao, J., Xie, S. Q., Das, R., \& Zhu, G. L. (2014). Control strategies for effective robot assisted gait rehabilitation: The state of art and future prospects. Medical Engineering \& Physics, 36(12), 1555-1566.

Carretero JA, Podhorodeski, RP, Nahon, MA, \& Gosselin, CM. (2000). Kinematic analysis and optimization of a new three degree of freedom spatial parallel manipulator. ASME Journal of Mechanical Design, 122(1), 17-24.

Cazalilla, J.I., Valles, M., Mata, V., Diaz-Rodriguez, M., Valera, A. (2014). Adaptive control of a 3-DOF parallel manipulator considering payload handling and relevant parameter models. Robotics and Computer-Integrated Manufacturing, 30(5), 468477.

Cetinkunt, S. (2015). Mechatronics with Experiments. John Wiley \& Sons. 
Codourey, A., \& Burdet, E. (1997). A body-oriented method for finding a linear form of the dynamic equation of fully parallel robots. In Proceedings of IEEE International Conference on Robotics and Automation, vol. 2, pp. 1612-1618.

Díaz, I., Gil, J. J., \& Sánchez, E. (2011). Lower-limb robotic rehabilitation: literature review and challenges. Journal of Robotics, 2011, 1-11.

Díaz-Rodríguez, M., Iriarte, X., Mata, V., \& Ros, J. (2009). On the experiment design for direct dynamic parameter identification of parallel robots. Advanced Robotics, 23(3), 329-348.

Díaz-Rodríguez, M., Mata, V., Valera, A., \& Page, A (2010). A methodology for dynamic parameters identification of 3-DOF parallel robots in terms of relevant parameters. Mechanism and Machine Theory, 45(9), 1337-1356.

Emken, J. L., Bobrow, J. E., \& Reinkensmeyer, D. J. (2005). Robotic movement training as an optimization problem: designing a controller that assists only as needed. In 9th Int. Conf. on Rehabilitation Robotics, pp. 307-312.

Farhat, N., Diaz, M. A., \& Mata, V. (2007), Dynamic parameter identification of parallel robots considering physical feasibility and nonlinear friction models. In 12th IFToMM World Congress.

Ferreira, C., Reis, L. P., \& Santos, C. P. (2016). Review of Control Strategies for Lower Limb Prostheses. In Second Iberian Robotics Conference, pp. 209-220.

Gautier, M., \& Khalil, W. (1988). On the identification of the inertial parameters of robots. In Proceedings of the 27th IEEE Conference on Decision and Control (pp. 22642269).

Gautier, M., Khalil, W., \& Restrepo, P. P. (1995). Identification of the dynamic parameters of a closed loop robot. In Proceedings of IEEE International Conference on Robotics and Automation (vol. 3, pp. 3045-3050).

Gautier, M. (1991). Numerical calculation of the base inertial parameters of robots. Journal of robotic systems, 8(4), 485-506.

Jamwal, P. K., Hussain, S., \& Xie, S. Q. (2015). Review on design and control aspects of ankle rehabilitation robots. Disability and Rehabilitation: Assistive Technology, 10(2), 93-101.

Janabi-Sharifi, F., Hayward, V. \& Chen, C.-S. J. (2000) Discrete-time adaptive windowing for velocity estimation, IEEE Trans. Control Syst. Technol., 8(6), 1003-1009. 
Janot, A., Gautier, M., Jubien, A., \& Vandanjon, P. O. (2014). Comparison between the CLOE Method and the DIDIM Method for Robots Identification. IEEE Transactions on Control Systems Technology, 22(5), 1935-1941.

Janot, A., Vandanjon, P. O., \& Gautier, M. (2016). A revised Durbin-Wu-Hausman test for industrial robot identification. Control Engineering Practice, 48, 52-62.

Jimenez-Fabian, R., \& Verlinden, O. (2012). Review of control algorithms for robotic ankle systems in lower-limb orthoses, prostheses, and exoskeletons. Medical Engineering \& Physics, 34(4), 397-408.

Khalil, W., Vijayalingam, A., Khomutenko, B., Mukhanov, I., Lemoine, P., \& Ecorchard, G. (2014). OpenSYMORO: An open-source software package for Symbolic Modelling of Robots. In Proceedings of the 2014 IEEE/ASME International Conference in Advanced Intelligent Mechatronics (pp. 1206-1211).

Klafter, R.D., Chmielewski, T.A., \& Negin, M. (1989). Robotics Engineering - An Integrated Approach. Englewood Cliffs, Prentice-Hall Int. Editions.

Kwakernaak, H., \& Sivan, R. (1972). Linear Optimal Control Systems, New York, Wiley.

Marchal-Crespo, L., \& Reinkensmeyer, D. J. (2009). Review of control strategies for robotic movement training after neurologic injury. Journal of Neuroengineering and Rehabilitation, 6(1), 20 ,

Meng, W., Liu, Q., Zhou, Z., Ai, Q., Sheng, B., \& Xie, S. S. (2015). Recent development of mechanisms and control strategies for robot-assisted lower limb rehabilitation. Mechatronics, 31,132-145.

Page, A., Candelas, P. \& Belmar, F. (2006). On the use of local fitting techniques for the analysis of physical dynamic systems. European Journal of Physics, 27(2), 273-279.

Pham, C. M., \& Gautier, M. (1991). Essential parameters of robots. In Proceedings of the 30th IEEE Conference on Decision and Control (pp. 2769-2774).

Raibert, H., \& Craig, J.J. (1981). Hybrid position/force control of manipulators, ASME J. Dynamic Systems, Measurement, and Control. 102, 126-133.

Ramsay, James O., \& Silverman, B. W. (2005). Functional Data Analysis. Springer.

Saglia, J. A., Tsagarakis, N. G., Dai, J. S., \& Caldwell, D. G. (2013). Control strategies for patient-assisted training using the ankle rehabilitation robot (ARBOT), IEEE/ASME Transactions on Mechatronics, 18(6), 1799-1808. 
Stan, S.D., Balan, R., Maties, V., \& Rad, C. (2009). Kinematics and fuzzy control of ISOGLIDE3 medical parallel robot. Mechanika, 1(75), 62-66.

Vallés, M., Díaz-Rodríguez, M., Valera, A., Mata, V., \& Page, A. (2012). Mechatronic development and dynamic control of a 3-DOF parallel manipulator. Mechanics based design of structures and machines, 40(4), 434-452.

Vallés, M., Cazalilla, J., Valera, Á., Mata, V., Page, Á., \& Díaz-Rodríguez, M. (2015). A 3PRS parallel manipulator for ankle rehabilitation: towards a low-cost robotic rehabilitation. Robotica, 1-19.

Vallés, M., Cazalilla, J. I., Valera, Á., Mata, V., \& Page, Á. (2013). Implementación basada en el middleware OROCOS de controladores dinámicos pasivos para un robot paralelo. Revista Iberoamericana de Automática e Informática Industrial RIAI, 10(1), 96-103.

Wu, F.X., Zhang, W.J., Li, Q., \& Ouyang, P.R. (2002). Integrated design and PD control of high-speed closed-loop mechanisms. Journal Dynamics Measurements and Control, 124(4), 522-528.

Yang, C., Huang, Q., \& Han, J. (2012). Decoupling control for spatial six-degree-offreedom electro-hydraulic parallel robot. Robotics and Computer-Integrated Manufacturing, 28(1), 14-23.

Yoon, J., Ryu, J., \& Lim, K.B. (2006). Reconfigurable Ankle Rehabilitation Robot for Various Exercises. Journal of Robotic Systems, 22 (1), 15-33. 


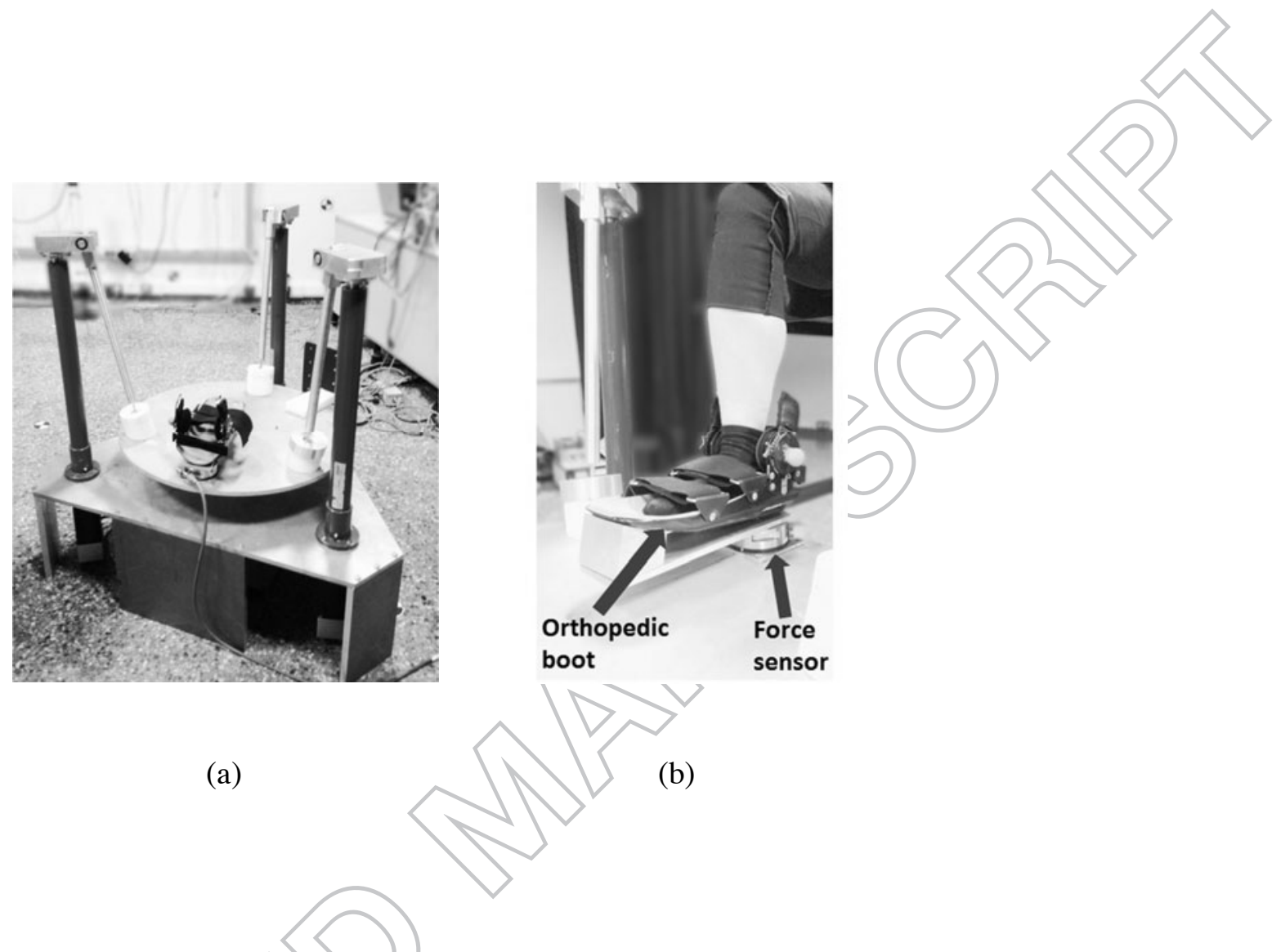

Figure. 1: Electromechanical Lower-limb Rehabilitation System 


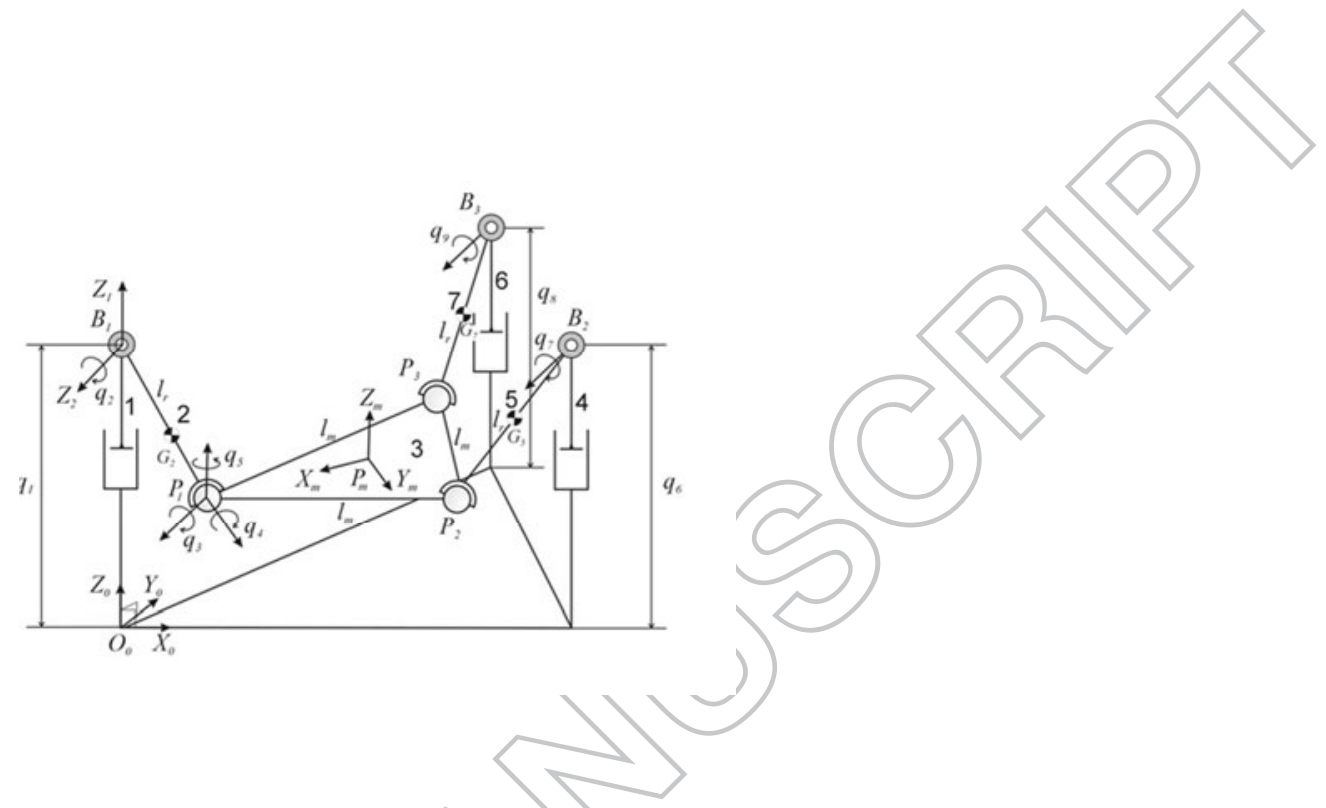

Figure 2. Kinematic diagram of the 3-PRS parallel robot, type of joints and generalized coordinates. 

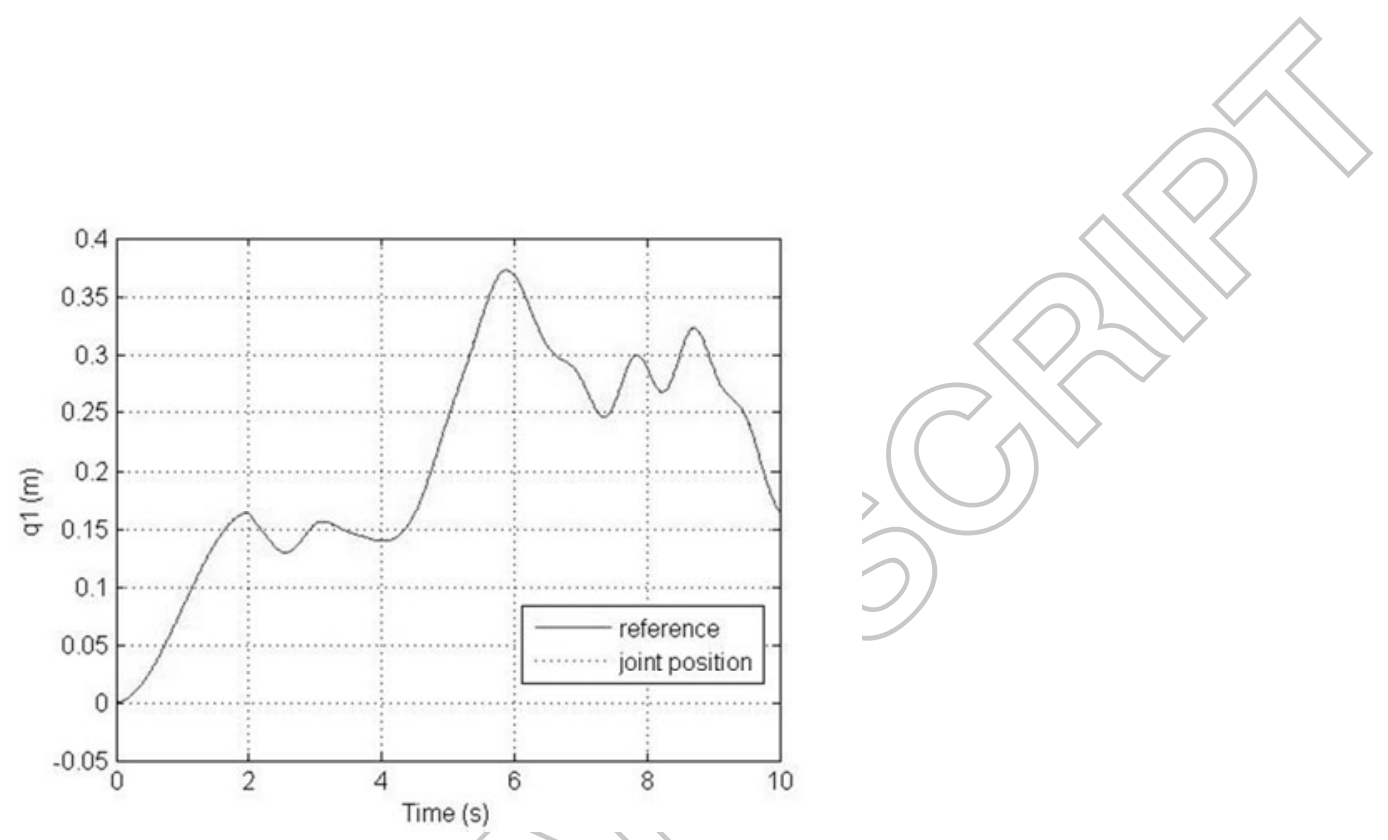

Figure. 3: Position for the q1 joint of the simulated PKM.

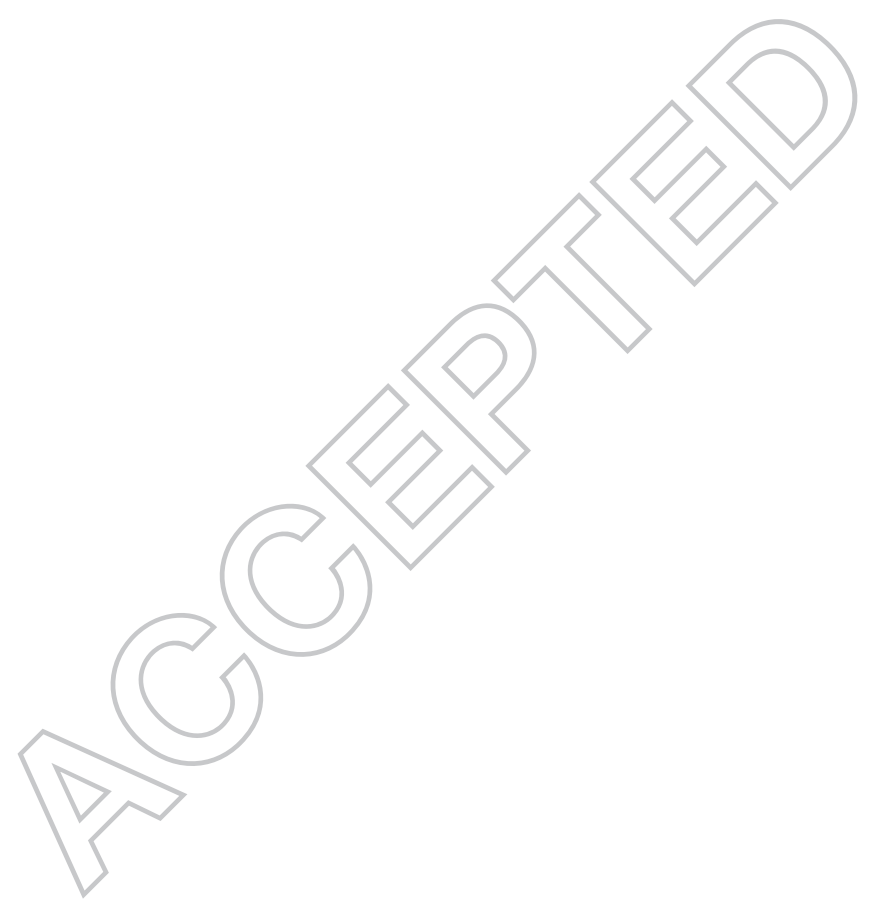



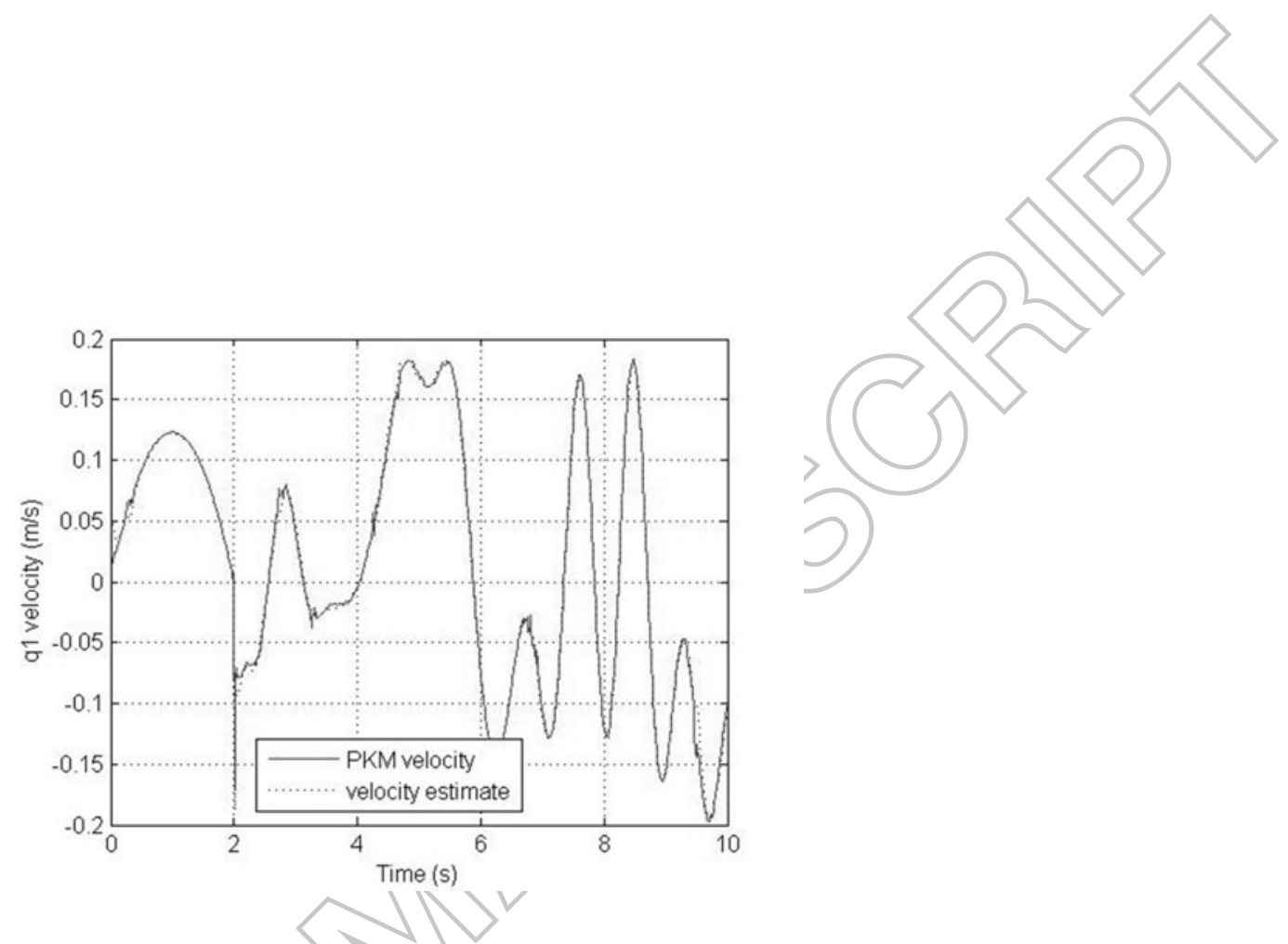

Figure. 4: Velocity for the q1 joint of the simulated PKM.

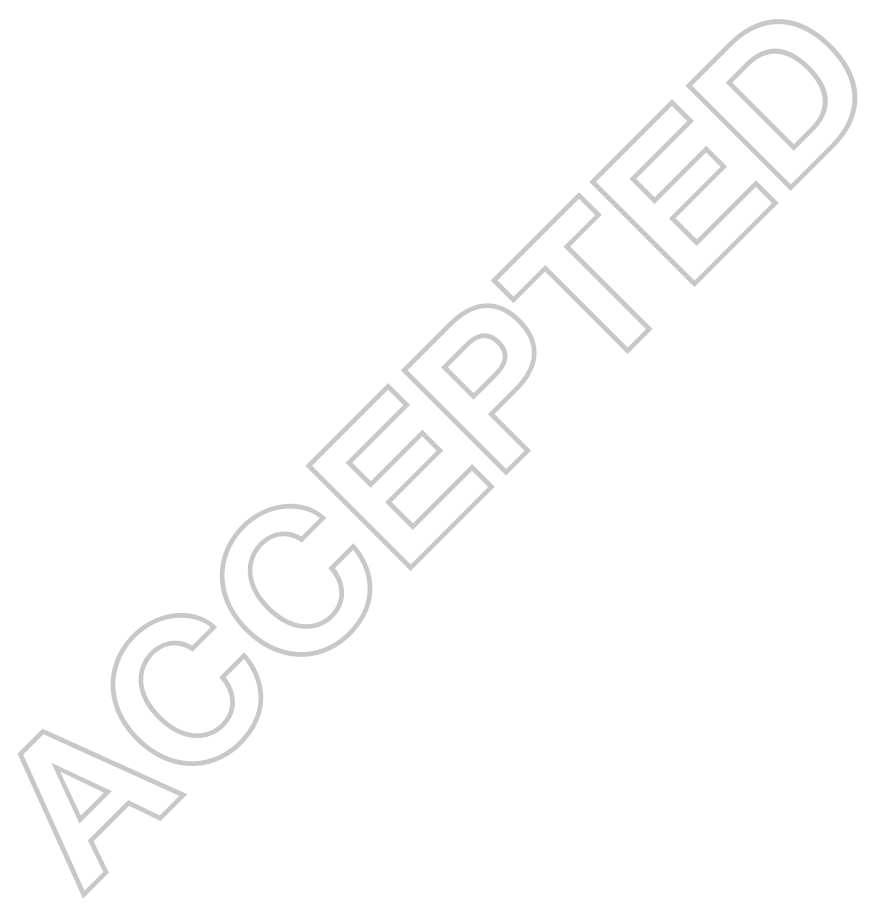



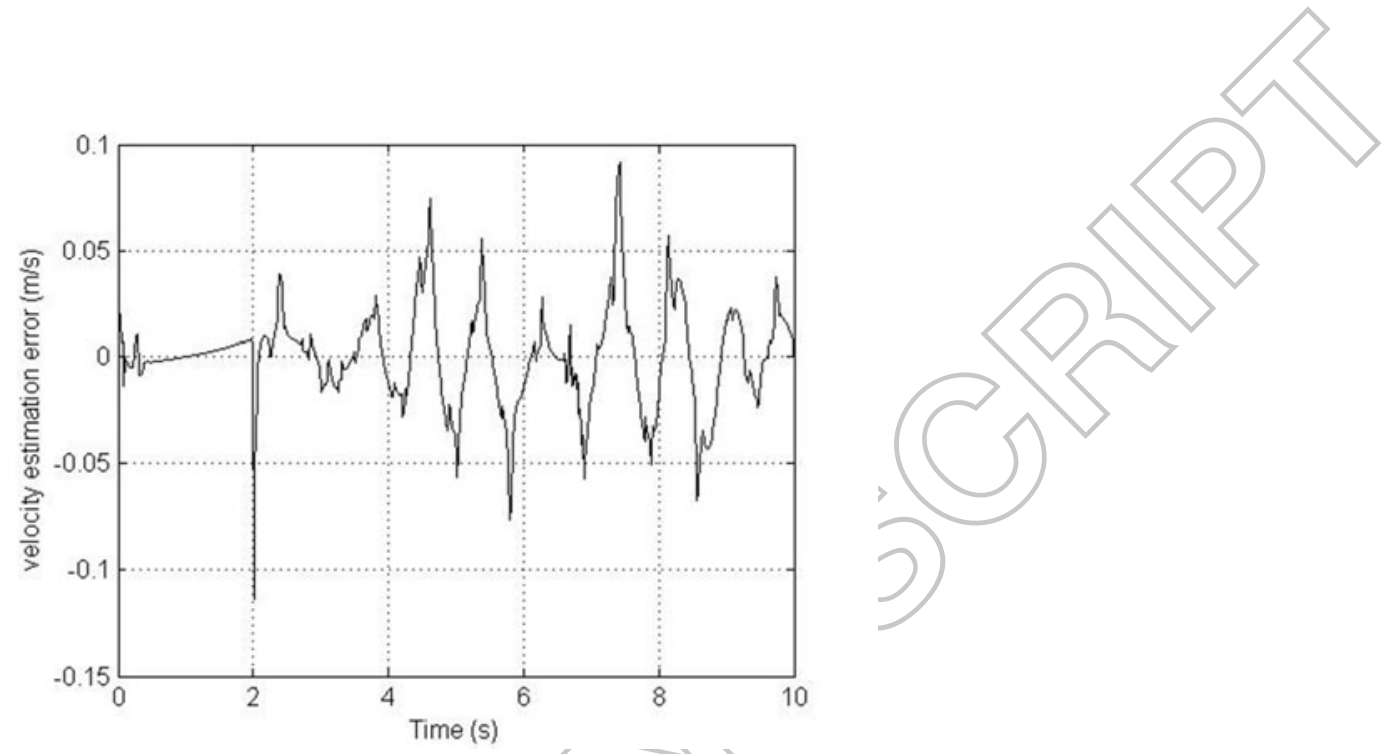

Figure. 5: Velocity estimation error 

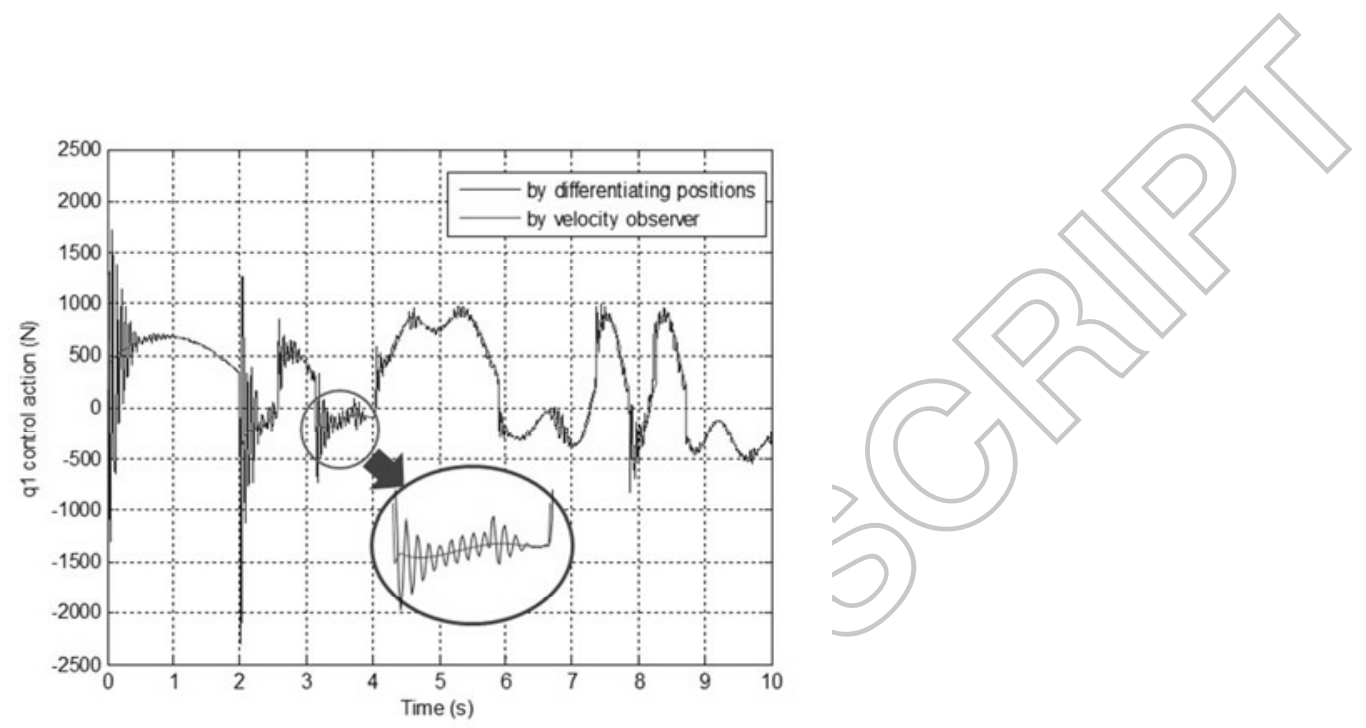

Figure. 6: Torques obtained in a simulation when using velocity estimation by the numerical method and using the controller observer.

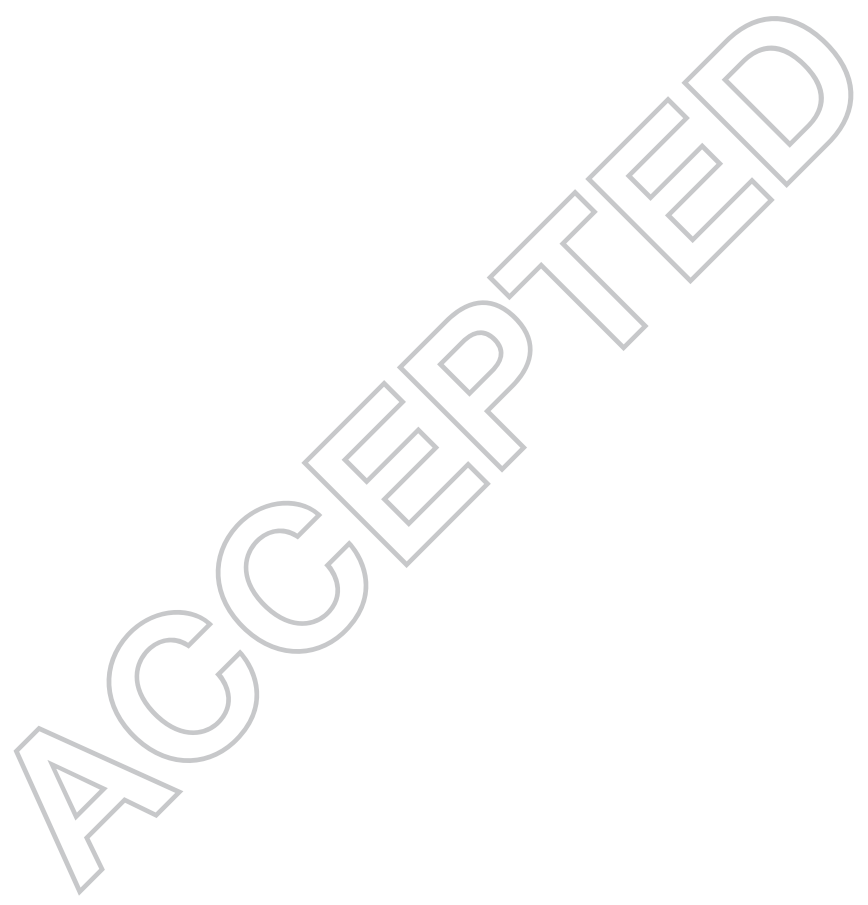



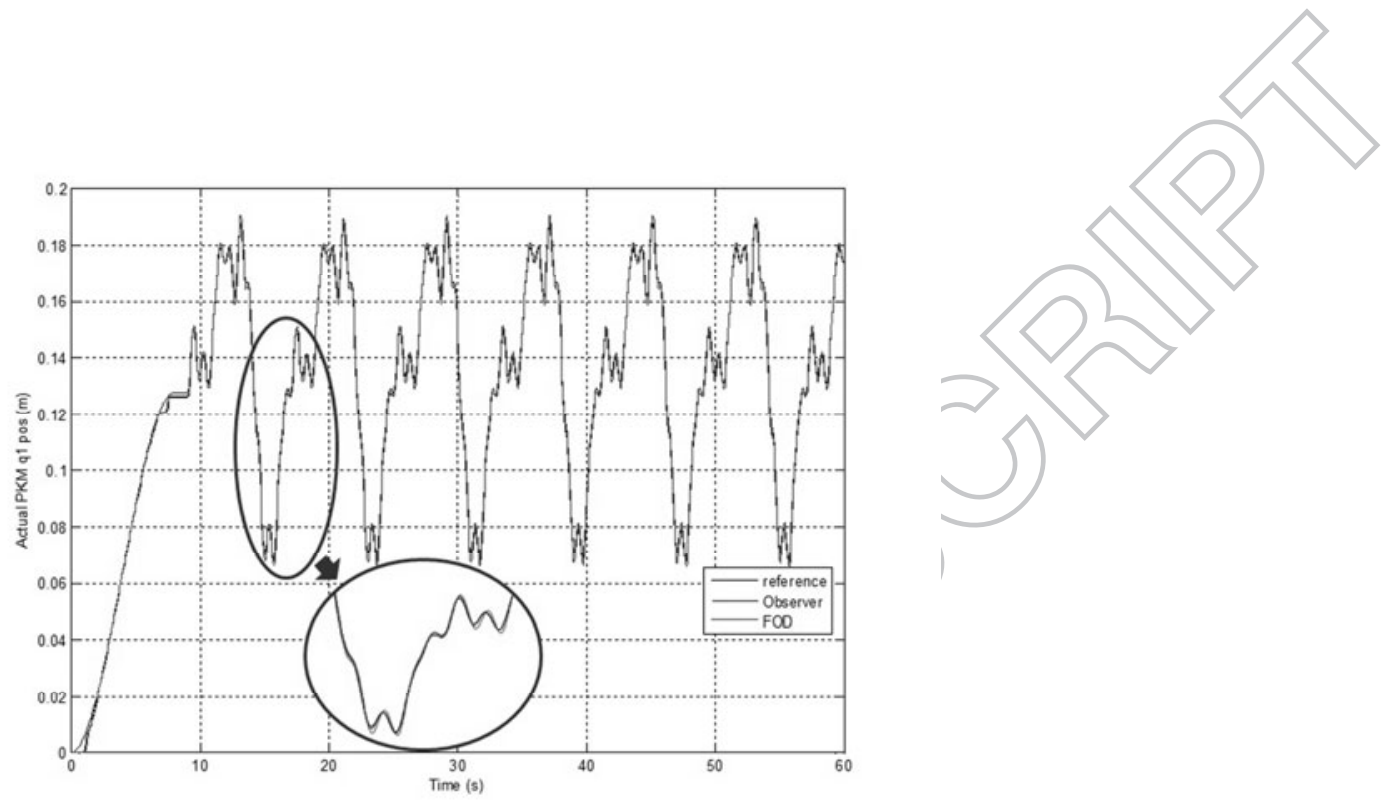

Figure. 7: Position for the $q_{1}$ joint of the actual PKM.

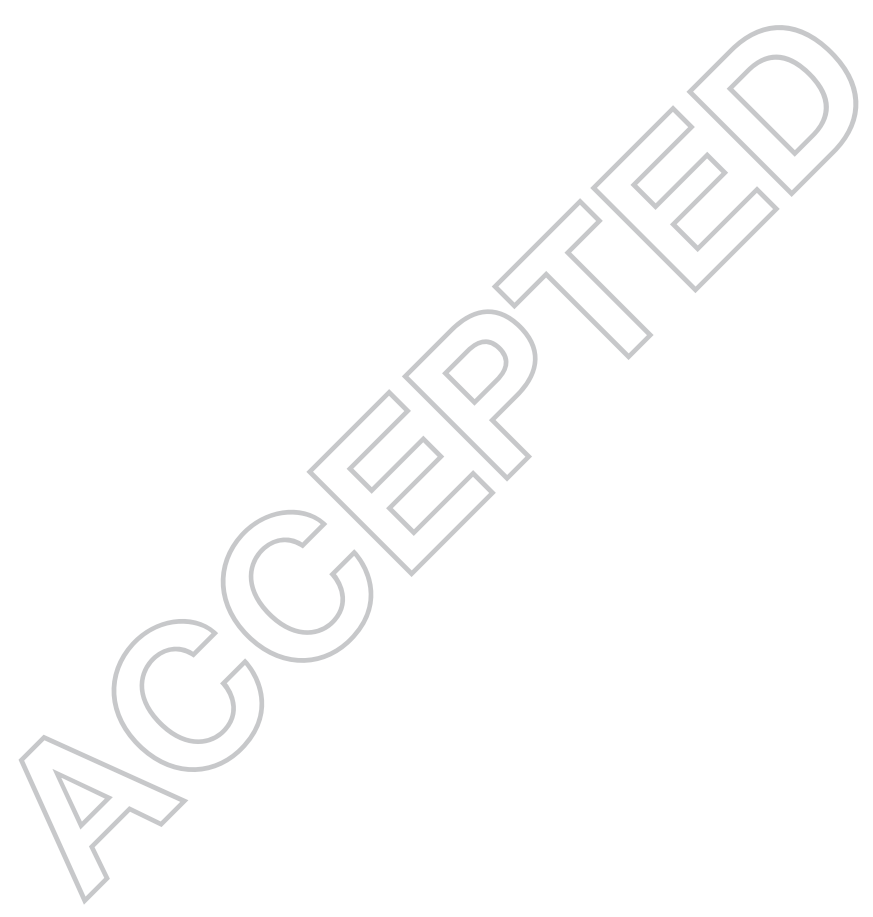



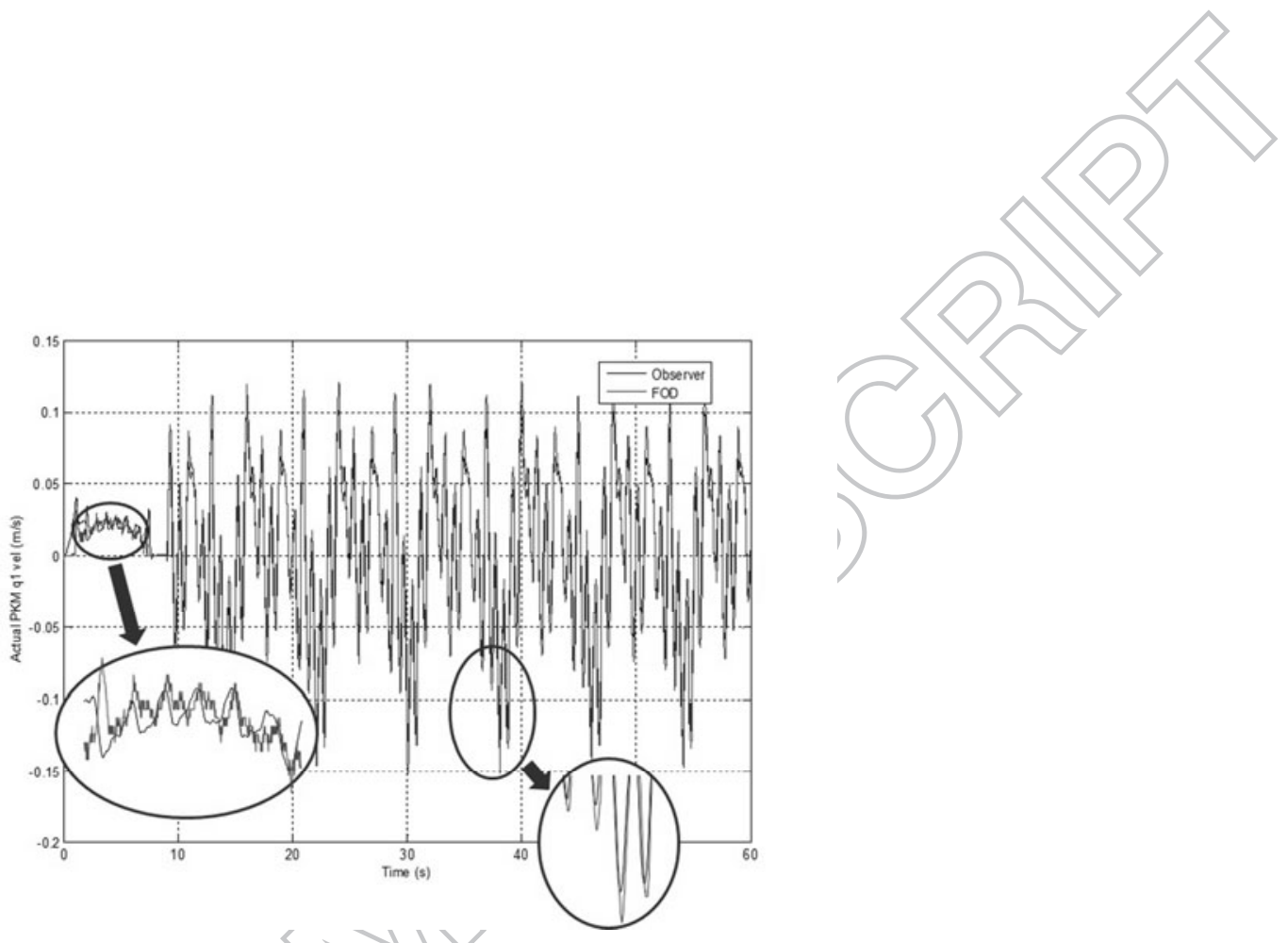

Figure. 8: Velocity for the $q_{1}$ joint of the actual PKM.

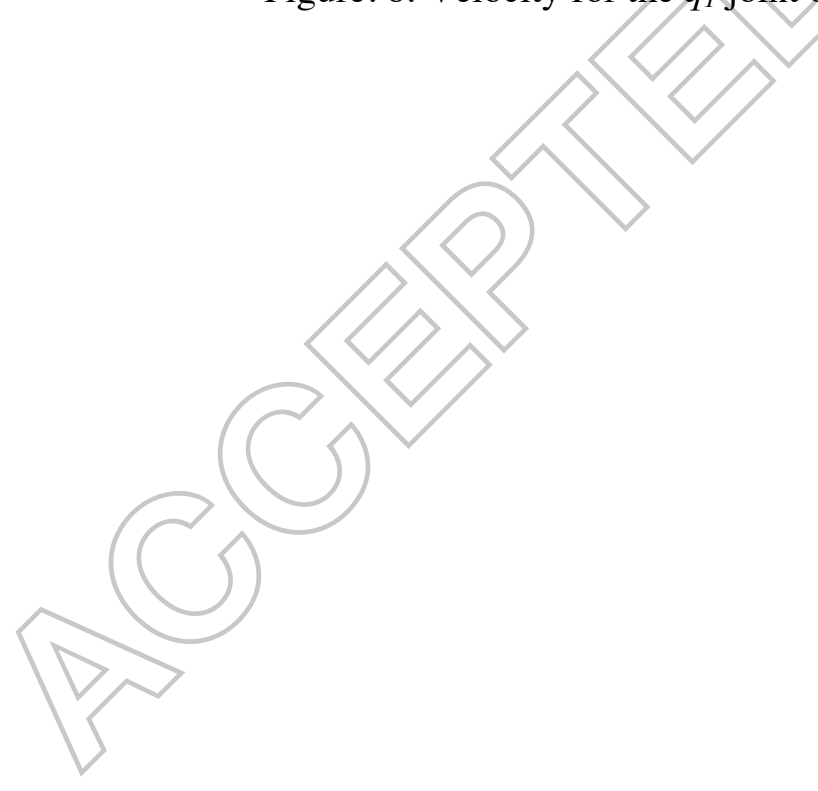




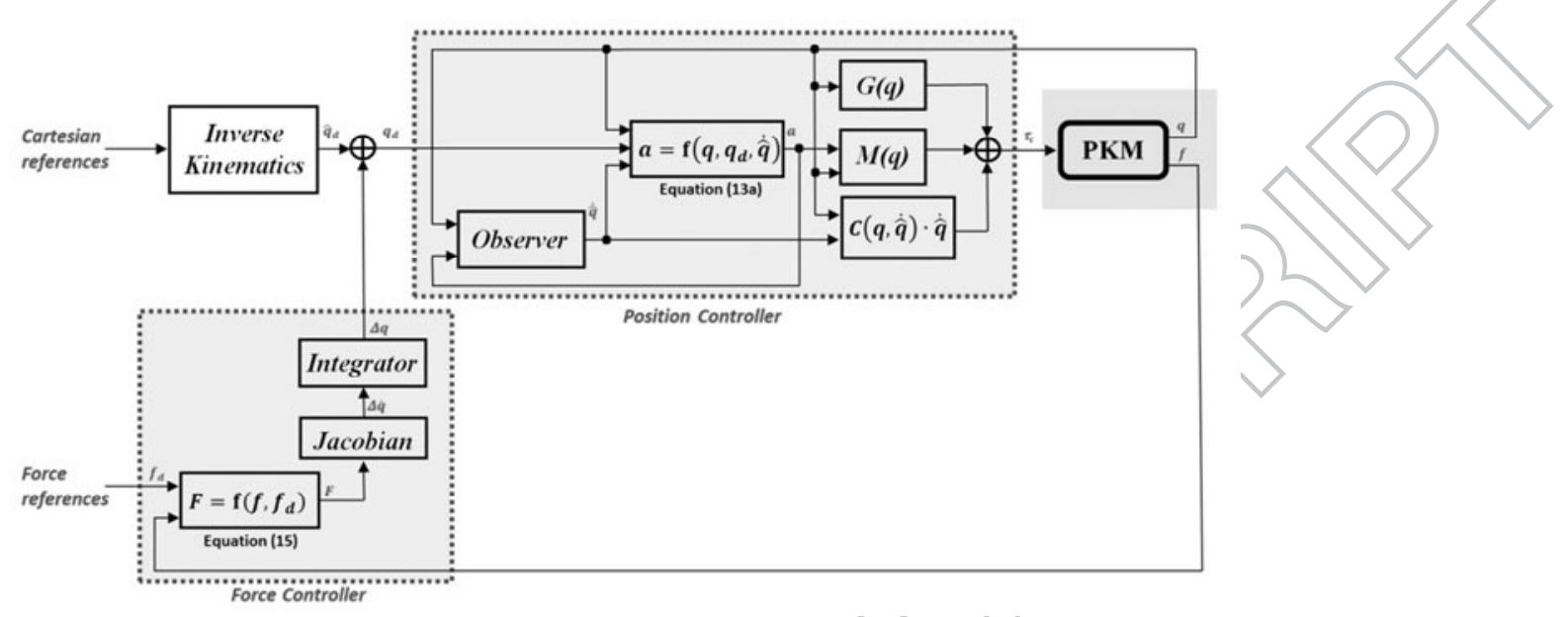

Figure. 9: Hybrid force/position control scheme. 

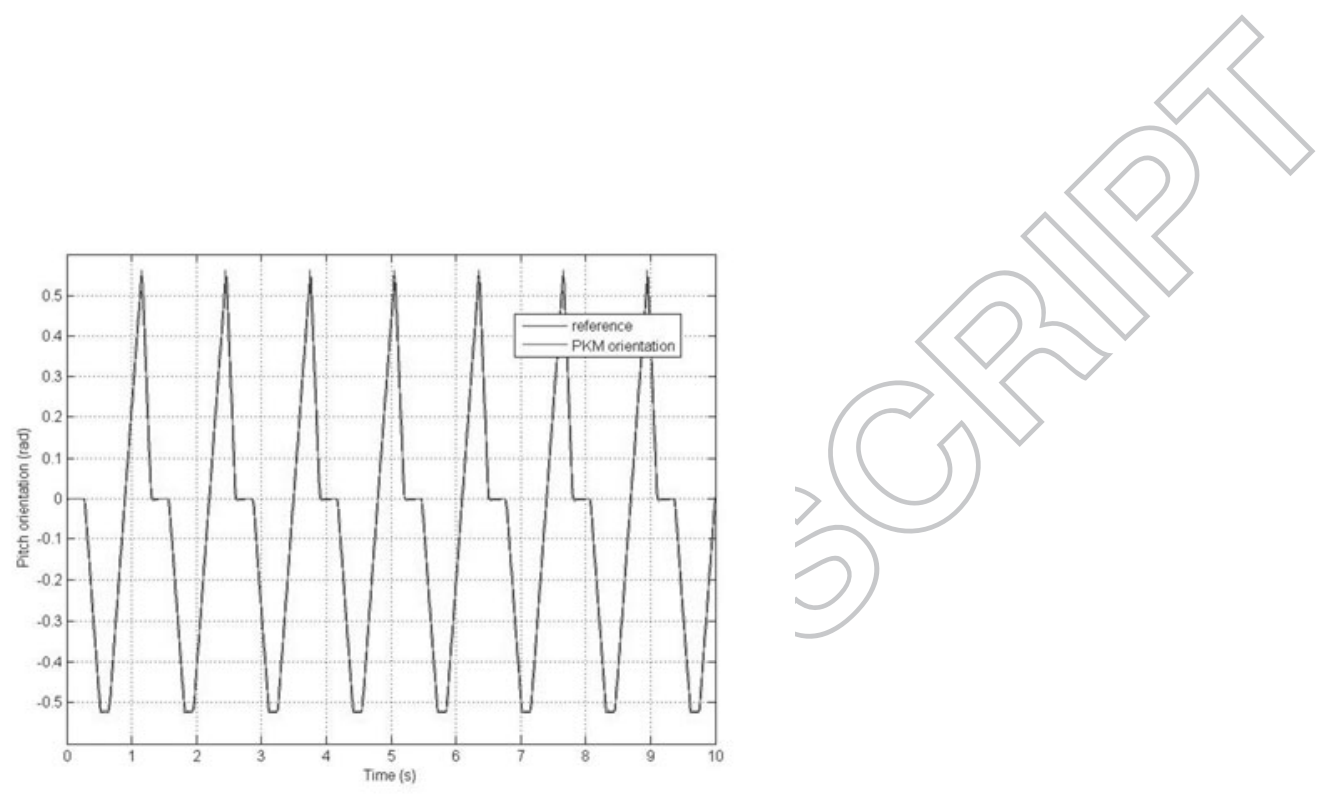

Figure. 10: Pitch orientation for mobile platform.

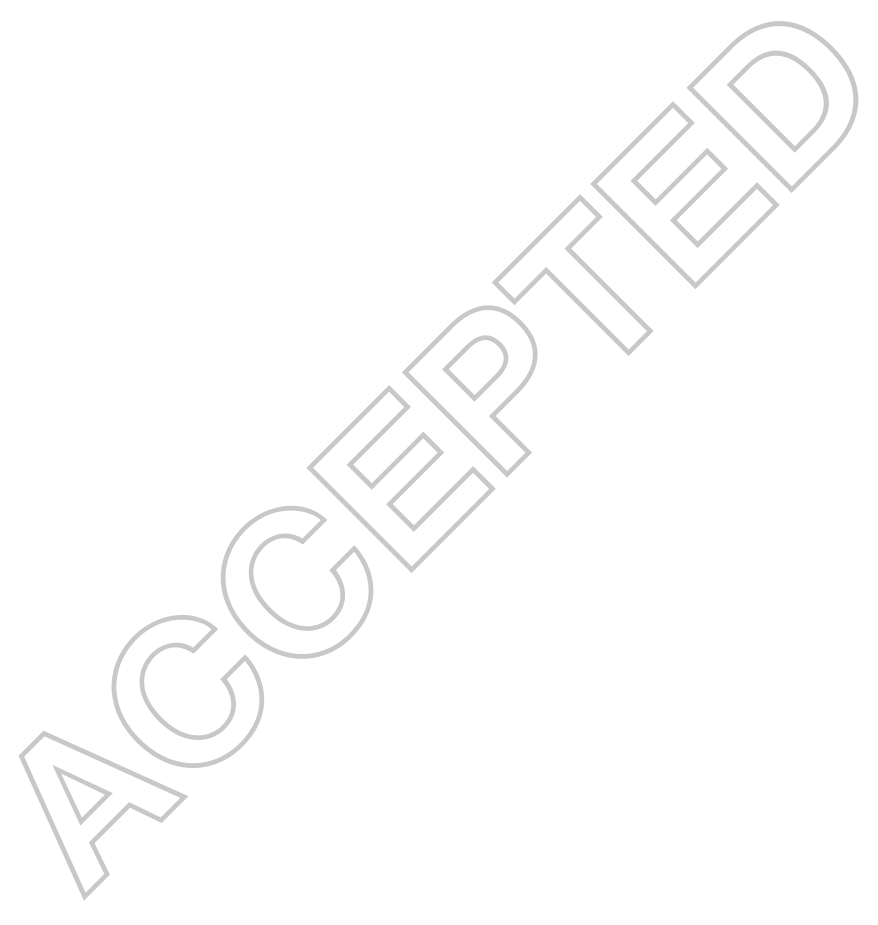



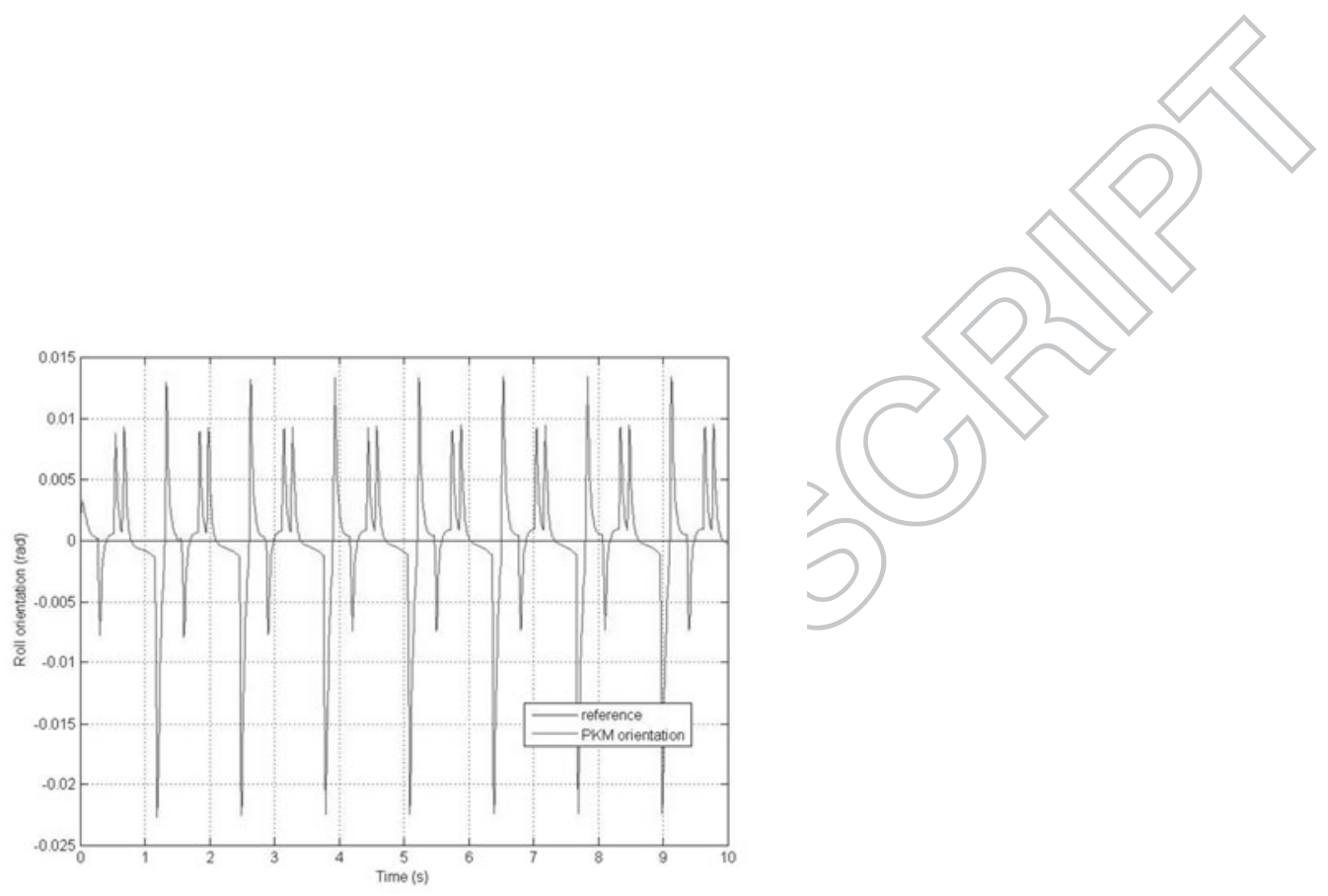

Figure. 11: Roll orientation for mobile platform

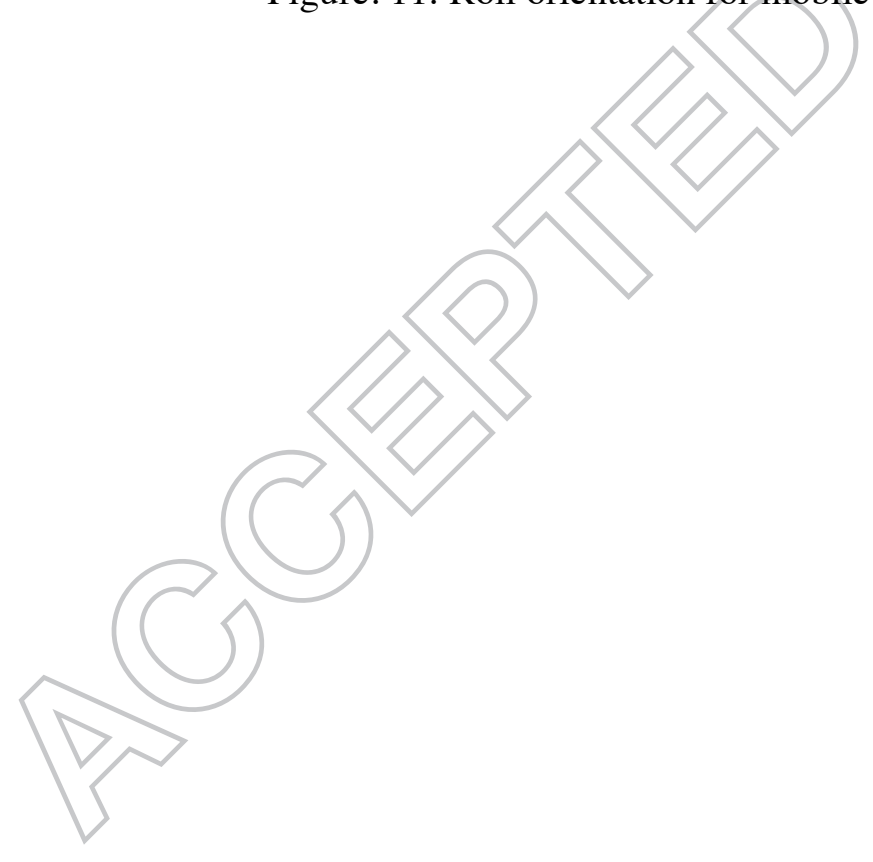




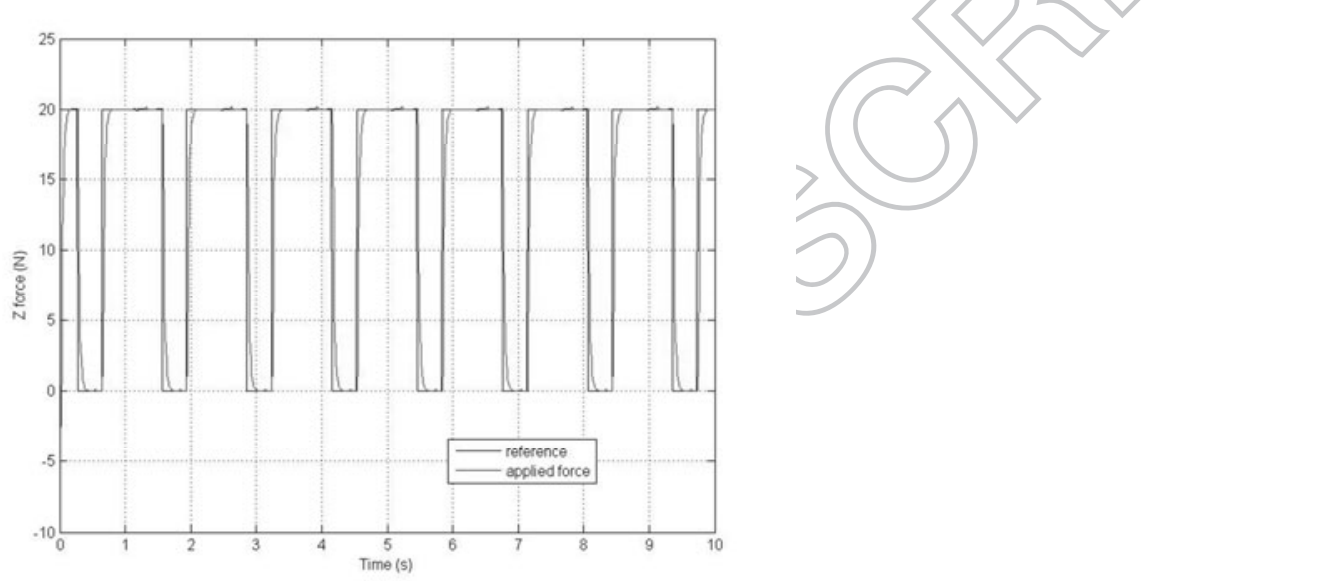

Figure. 12: Force applied by the platform.
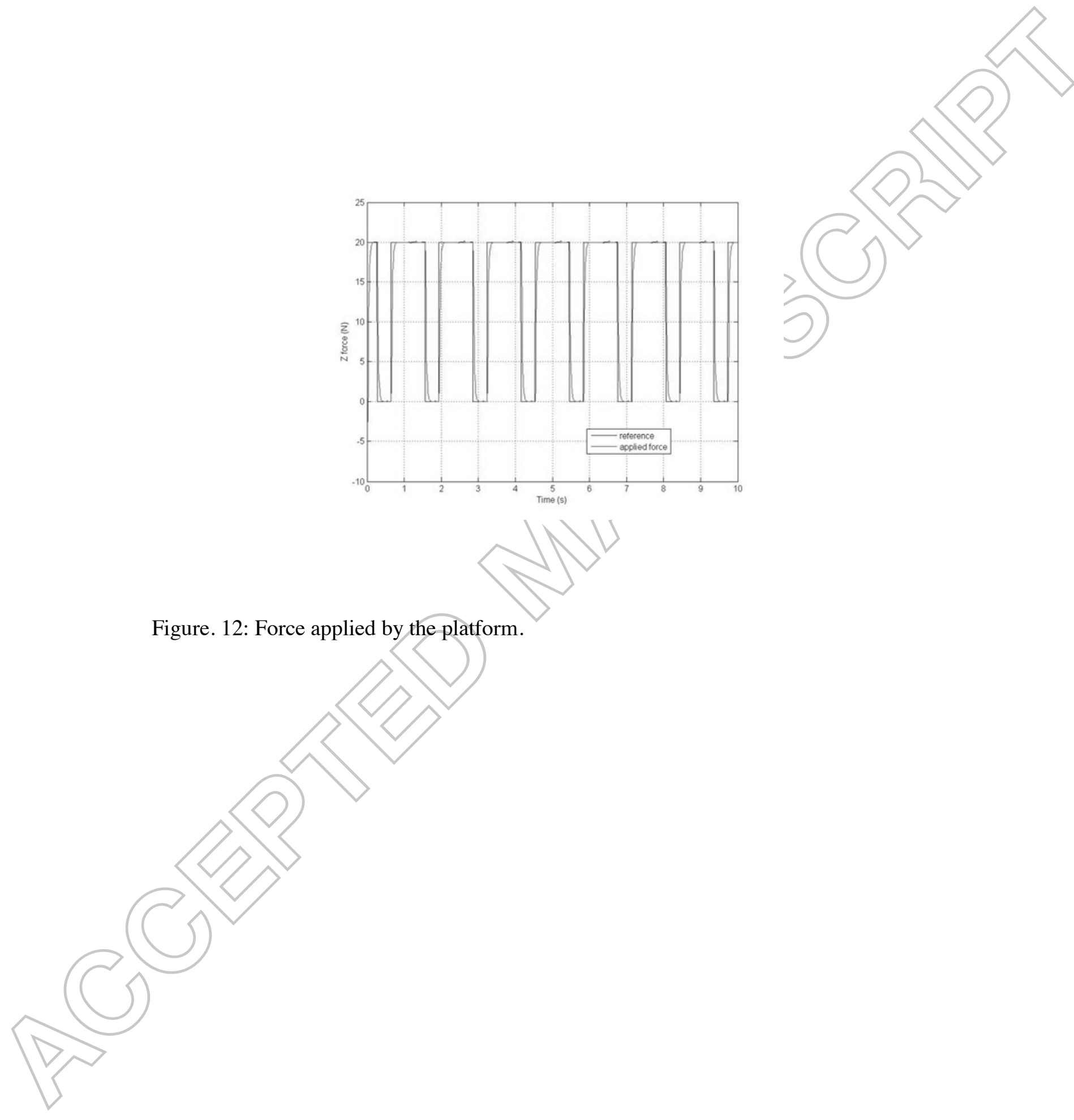

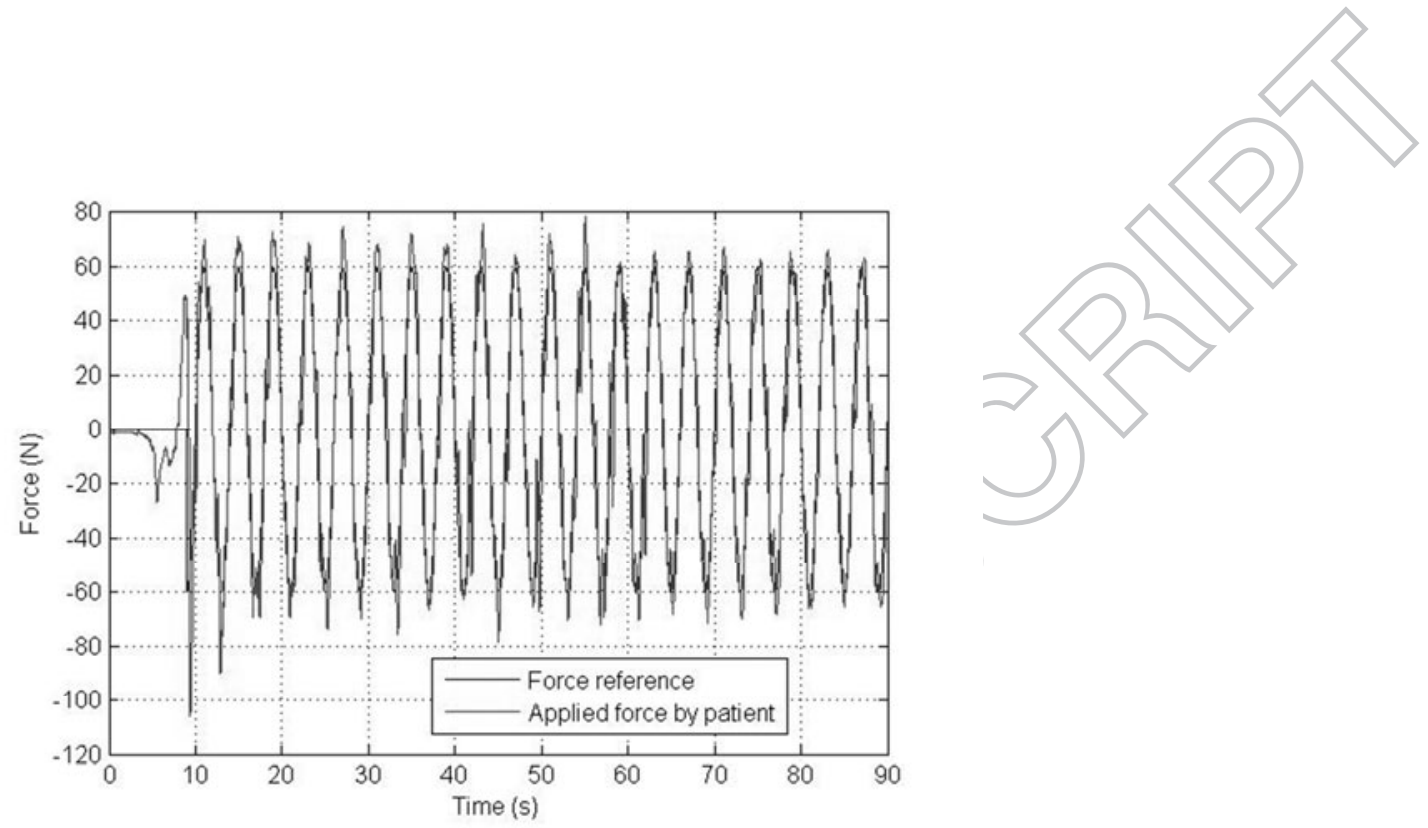

Figure. 13: Reference and force applied in the exercise.

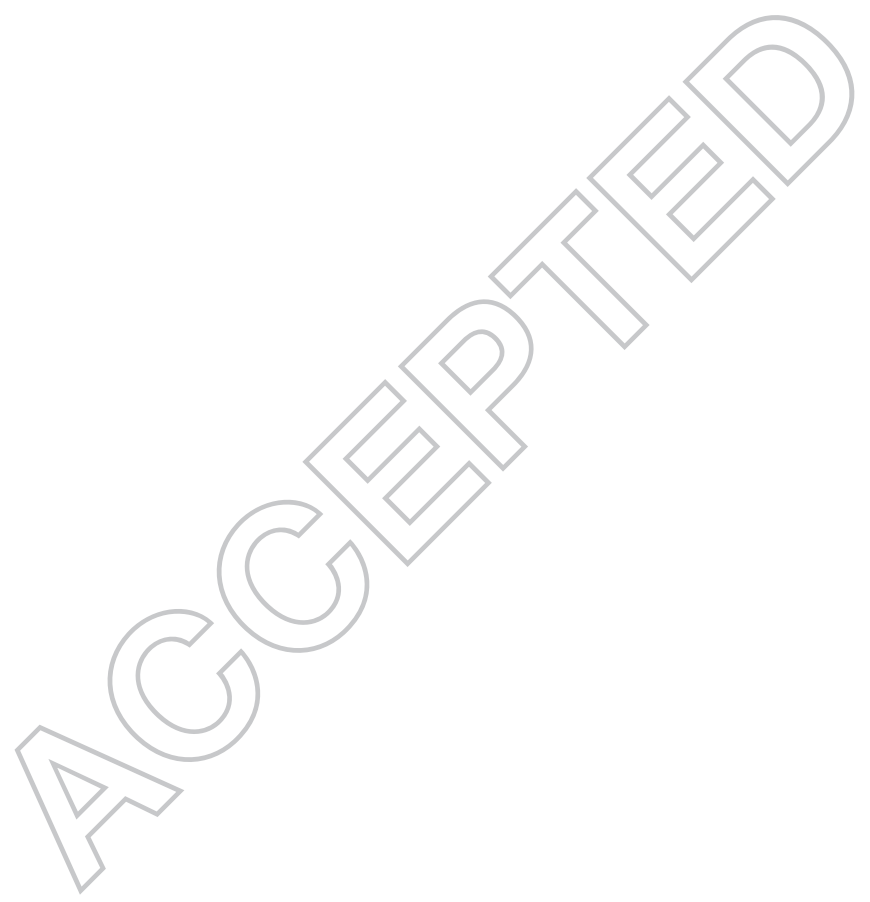



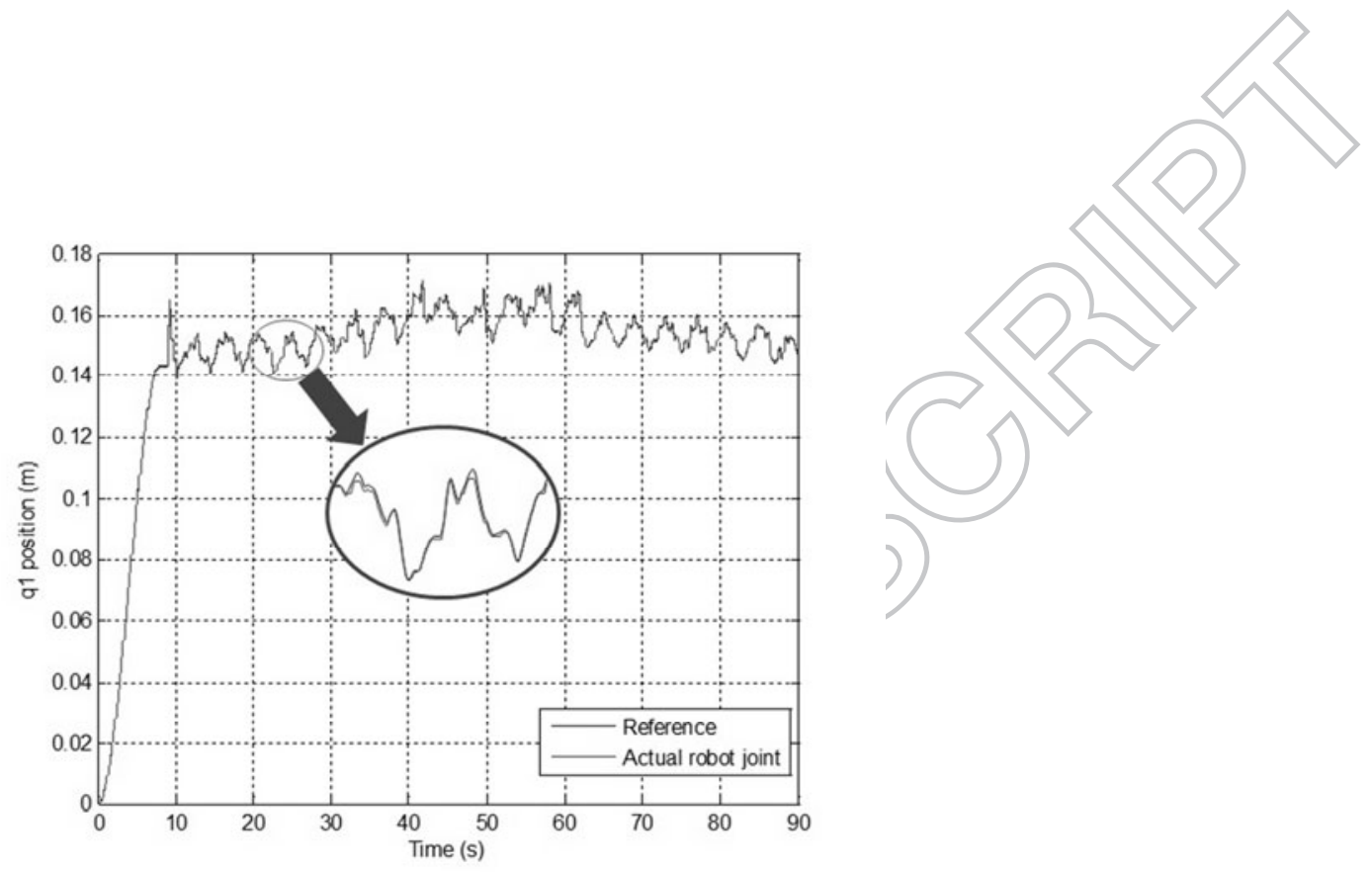

Figure. 14: Position of the q1 joint of the actual PKM for the rehabilitation exercise.

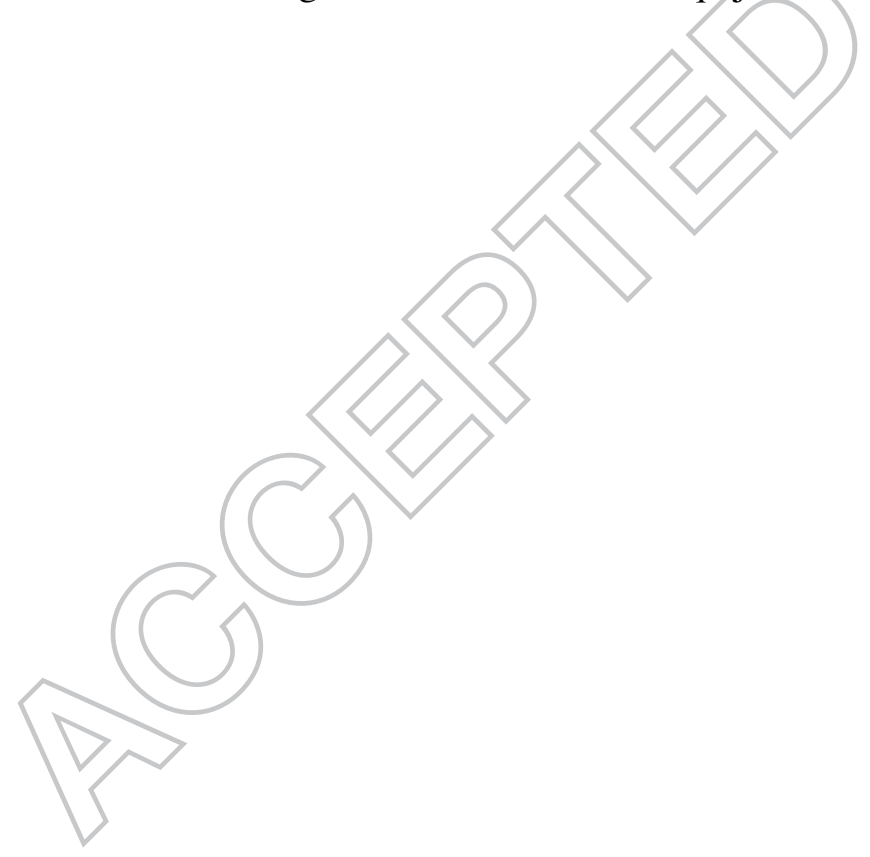



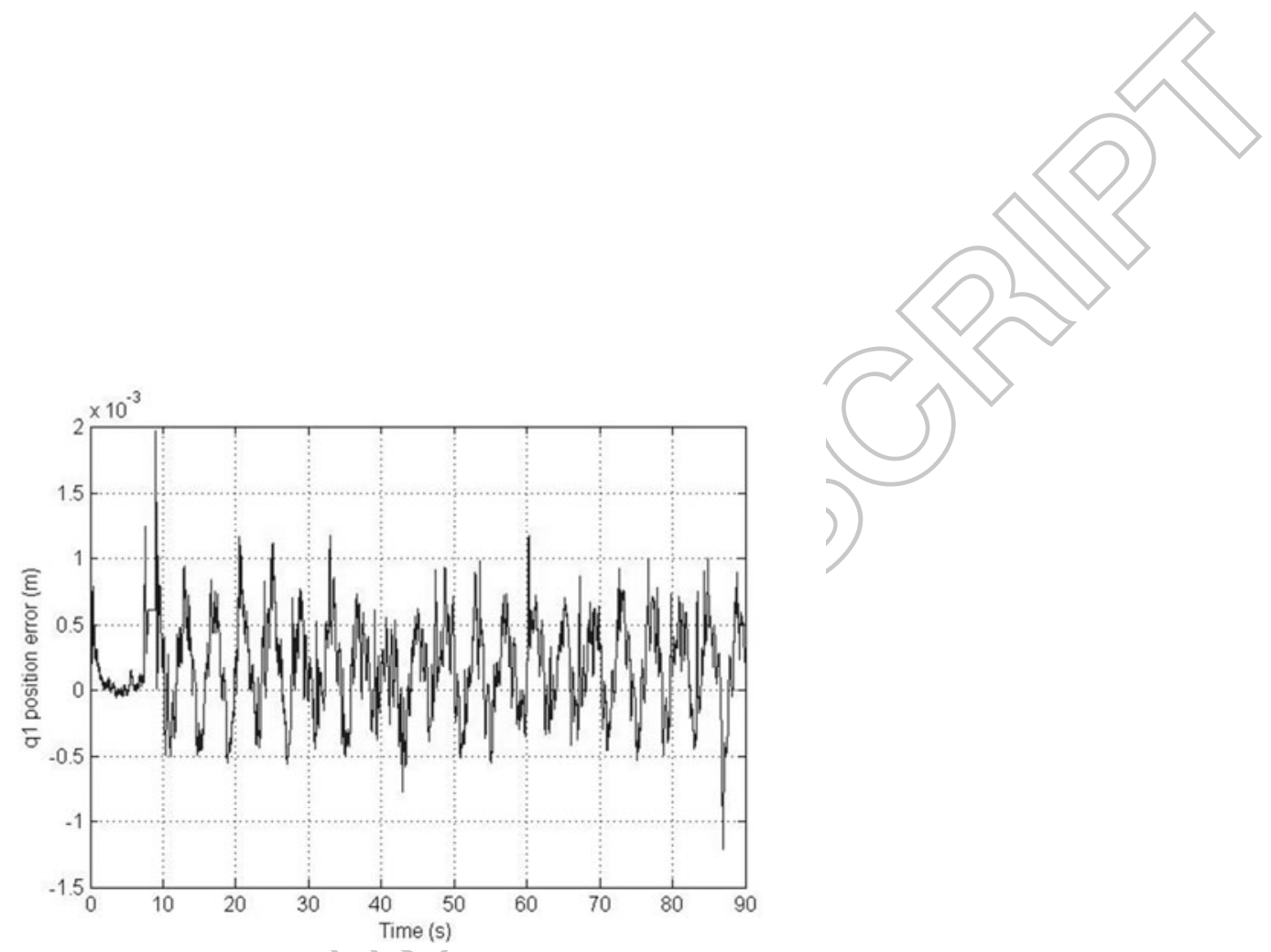

Figure. 15: Joint position error for the rehabilitation task of the actual system.

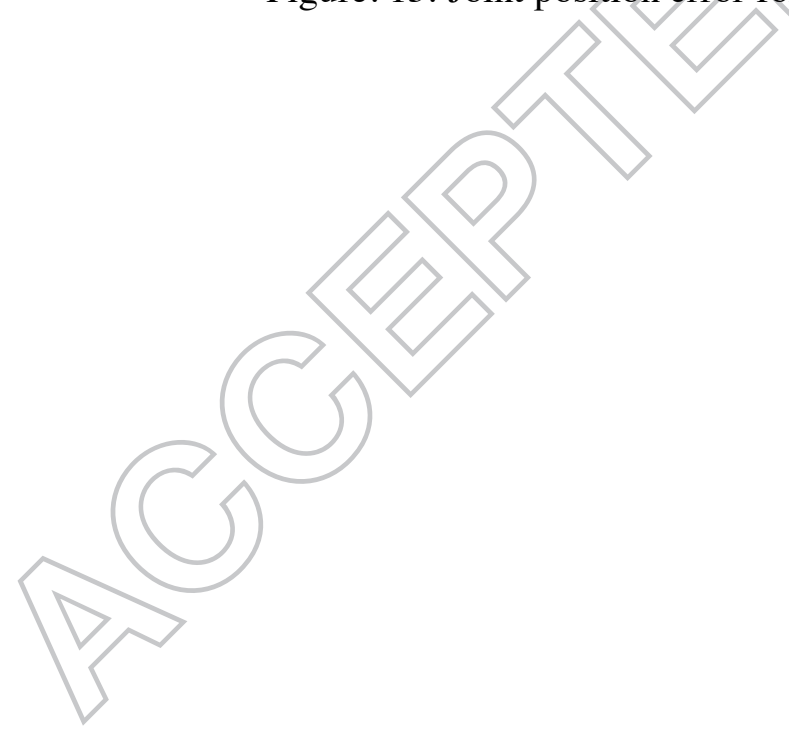


Table 1. Base parameters considering that all the legs have identical geometries.

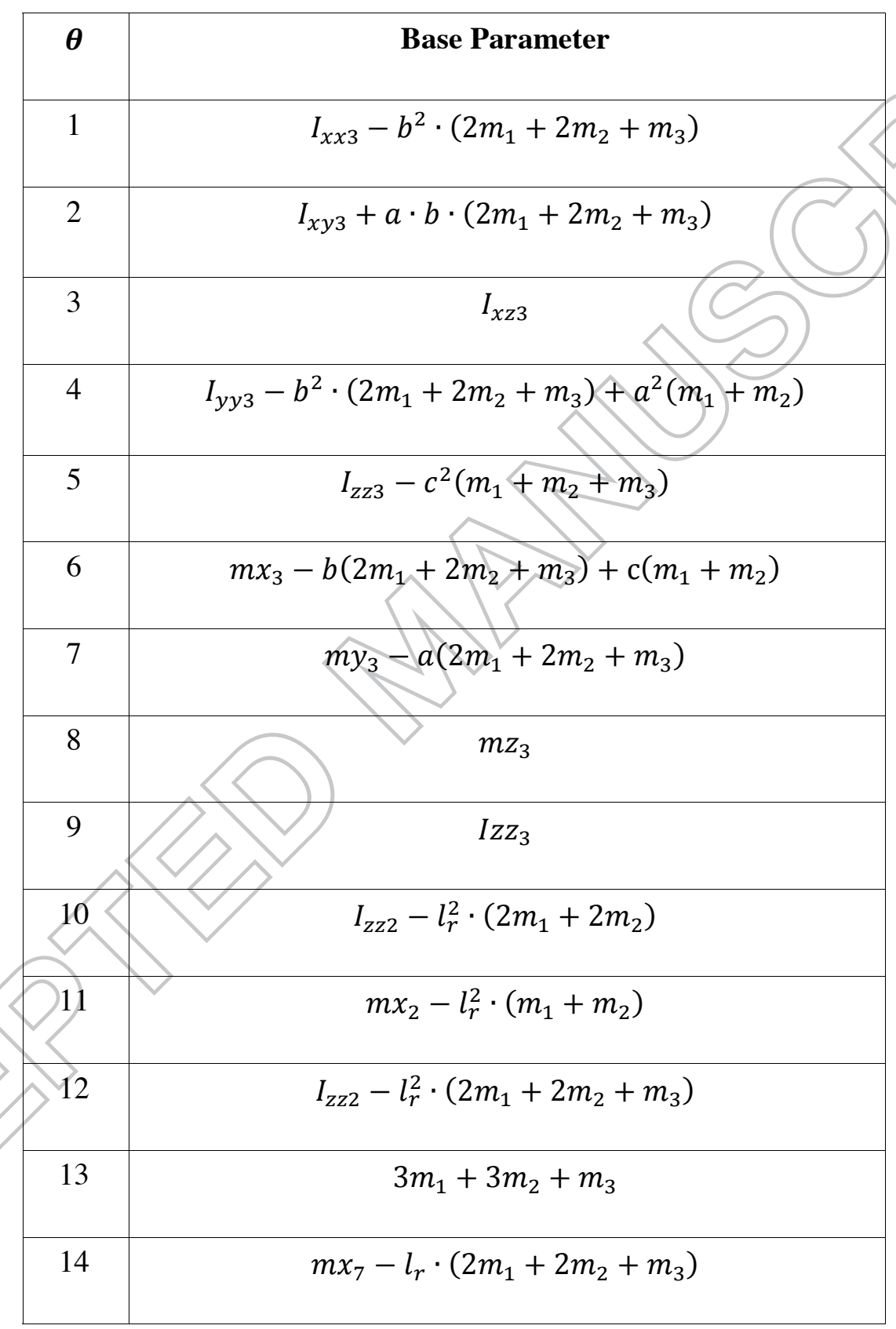


Table 2. Performance comparison between velocity estimations.

\begin{tabular}{|l|l|l|c|c|c|}
\cline { 3 - 5 } \multicolumn{2}{c|}{} & \multicolumn{2}{c|}{ FOD estimation } & \multicolumn{2}{c|}{ Observer estimation } \\
\hline \multirow{3}{*}{ Variable } & $\begin{array}{l}\text { Comparison } \\
\text { Parameter }\end{array}$ & Absolute & \% of range & Absolute & \% of range \\
\hline \multirow{2}{*}{ Position q1 } & Mean of error & $-3.2 \times 10^{-4}$ & -0.26 & $-2.9 \times 10^{-4}$ & -0.24 \\
\cline { 2 - 6 } & Std of error & $2.0 \times 10^{-3}$ & 1.68 & $0.93 \times 10^{-3}$ & 0.78 \\
\cline { 2 - 6 } & $\operatorname{Lag}\left(^{\circ}\right)$ & 0.88 & - & 0.41 & - \\
\hline \multirow{2}{*}{ Velocity } & Mean of error & $1.4 \times 10^{-5}$ & $<0.01$ & $1.0 \times 10^{-4}$ & $<0.05$ \\
\cline { 2 - 6 } & Std of error & 0.016 & 7.29 & 0.004 & 1.98 \\
\cline { 2 - 6 } & $\operatorname{Lag}\left(^{\circ}\right)$ & 16.8 & - & 4.9 & \\
\hline
\end{tabular}

